\title{
ADJOINT LIFTS AND MODULAR ENDOMORPHISM ALGEBRAS
}

\author{
BY \\ Debargha BAnerJeE \\ Mathematical Sciences Institute, Australian National University \\ Canberra, ACT 0200, Australia \\ e-mail:debargha.banerjee@anu.edu.au
}

AND

EknAth Ghate

School of Mathematics, TIFR, Homi Bhabha Road

Mumbai 400005, India

e-mail: eghate@math.tifr.res.in

\begin{abstract}
We prove that the ramification of the endomorphism algebra of the Grothendieck motive attached to a non-CM cuspform of weight two or more is completely determined by the slopes of the adjoint lift of this form, when the slopes are finite. We treat all places of good and bad reduction, answering a question of Ribet about the Brauer class of the endomorphism algebra in the finite slope case.
\end{abstract}

\section{Introduction}

Let $f=\sum_{n=1}^{\infty} a_{n} q^{n}$ be a primitive non-CM cusp form of weight $k \geq 2$, level $N \geq 1$ and character $\epsilon$, and let $M_{f}$ be the motive attached to $f$. If $f$ has weight $2, M_{f}$ is the abelian variety attached to $f$ by Shimura [Sh71], and for weights larger than $2, M_{f}$ is the Grothendieck motive attached to $f$ by Scholl in [Sc90]. In all cases, $M_{f}$ is a pure motive of rank 2 , weight $k-1$, with coefficients in the

Received August 4, 2011 and in revised form December 2, 2011 
Hecke field $E=\mathbb{Q}\left(a_{n}\right)$ of $f$. Let $\operatorname{End}\left(M_{f}\right)$ denote the ring of endomorphisms of $M_{f}$ defined over $\overline{\mathbb{Q}}$ and let

$$
X_{f}=\operatorname{End}\left(M_{f}\right) \otimes_{\mathbb{Z}} \mathbb{Q}
$$

be the $\mathbb{Q}$-algebra of endomorphisms of $M_{f}$. One knows that $X_{f}$ is a central simple algebra over a subfield $F$ of $E$, and that the class of $X_{f}$ in the Brauer group $\operatorname{Br}(F)$ of $F$ is 2-torsion. Ribet has remarked that it seems difficult to describe this class by pure thought. The goal of this paper is to give a complete description of the class of $X_{f}$ in terms of the slopes of a functorial lift of $f$, under a finiteness hypothesis on these slopes.

That $X_{f}$ is a central simple algebra over $F$ follows from an explicit structure theorem for $X_{f}$ which shows that $X_{f}$ is isomorphic to a crossed product algebra. Let $\Gamma \subset \operatorname{Aut}(E)$ be the group of extra twists of $f$. Recall that a pair $\left(\gamma, \chi_{\gamma}\right)$, where $\gamma \in \Gamma \subset \operatorname{Aut}(E)$ and $\chi_{\gamma}$ is an $E$-valued Dirichlet character, is called an extra twist for $f$ if $f^{\gamma}=f \otimes \chi_{\gamma}$, i.e., $a_{p}^{\gamma}=a_{p} \cdot \chi_{\gamma}(p)$, for all primes $p \nmid N$. Define the $E$-valued Jacobi sum 2-cocycle $c$ on $\Gamma$ by

$$
c(\gamma, \delta)=\frac{G\left(\chi_{\delta}^{-\gamma}\right) G\left(\chi_{\gamma}^{-1}\right)}{G\left(\chi_{\gamma \cdot \delta}^{-1}\right)} \in E,
$$

for $\gamma, \delta \in \Gamma$, where $G(\chi)$ is the usual Gauss sum attached to the character $\chi$. Let $X$ be the corresponding crossed product algebra defined by

$$
X=\bigoplus_{\gamma \in \Gamma} E \cdot x_{\gamma}
$$

where the $x_{\gamma}$ are formal symbols satisfying the relations

$$
\begin{aligned}
x_{\gamma} \cdot x_{\delta} & =c(\gamma, \delta) \cdot x_{\gamma \delta}, \\
x_{\gamma} \cdot e & =\gamma(e) \cdot x_{\gamma},
\end{aligned}
$$

for $\gamma, \delta \in \Gamma$ and $e \in E$. Clearly $X$ is a central simple algebra over $F$, the fixed field of $\Gamma$ in $E$. A fundamental result due to Momose [Mo81] and Ribet [Ri80] in weight 2 , and [BG04] (see also [GGQ05]) in higher weight, says that $X_{f} \cong X$. Moreover, $F \subset E$ is known to be the subfield generated by $a_{p}^{2} \epsilon^{-1}(p)$, for primes $p \nmid N$.

To study the Brauer class of $X=X_{f}$, the standard exact sequence from classfield theory

$$
0 \rightarrow{ }_{2} \operatorname{Br}(F) \rightarrow \oplus_{v} \operatorname{Br}\left(F_{v}\right) \rightarrow \mathbb{Z} / 2 \rightarrow 0
$$


where $v$ runs over all places of $F$, shows that it is enough to study the class of $X_{v}=X \otimes_{F} F_{v}$ in $\operatorname{Br}\left(F_{v}\right)$, for each place $v$. It is well known that ${ }_{2} \operatorname{Br}\left(F_{v}\right) \cong \mathbb{Z} / 2$, including if $v$ is infinite since $F$ is totally real, and $X_{v}$ is a matrix algebra over $F_{v}$ if the class of $X_{v}$ is trivial, and is a matrix algebra over a quaternion division algebra over $F_{v}$ if the class of $X_{v}$ is non-trivial. A theorem of Momose [Mo81] says that $X$ is totally indefinite if $k$ is even, and totally definite if $k$ is odd, giving complete information about the Brauer class at the infinite places $v$. When $v$ is a finite place, we shall prove in this paper that the class of $X_{v}$ in $\operatorname{Br}\left(F_{v}\right)$ is completely determined in terms of the parity of the slope at $v$ of the adjoint lift of $f$ (when this slope is finite).

According to Langlands principle of functoriality, given two reductive algebraic groups $\mathrm{H}$ and $\mathrm{G}$ over $\mathbb{Q}$ and a homomorphism between their $L$-groups $u:{ }^{L} \mathrm{H} \rightarrow{ }^{L} \mathrm{G}$, there should be a way to lift cuspidal automorphic representations $\pi$ of $H\left(\mathbb{A}_{\mathbb{Q}}\right)$ to cuspidal automorphic representation $\Pi$ of $G\left(\mathbb{A}_{\mathbb{Q}}\right)$, so that the Langlands $L$-functions of $\pi$ and $\Pi$ are related by the formula $L(s, \Pi, r)=L(s, \pi, r \circ u)$. In the case that $\mathrm{H}=\mathrm{GL}_{2}$ and $\mathrm{G}=\mathrm{GL}_{3}$, and $u$ is the adjoint map, it is (by now) a classical theorem of Gelbart and Jacquet [GJ78] that every cuspidal automorphic form $\pi$ on $\mathrm{GL}_{2}\left(\mathbb{A}_{\mathbb{Q}}\right)$ has a lift $\operatorname{ad}(\pi)$, called the Gelbart-Jacquet adjoint lift, to an automorphic representation of $\mathrm{GL}_{3}\left(\mathbb{A}_{\mathbb{Q}}\right)$. If the Satake parameters at an unramified prime $p$ of $\pi$ are $\alpha_{p}$ and $\beta_{p}$, then the Satake parameters of the adjoint lift $\operatorname{ad}(\pi)$ are $\frac{\alpha_{p}}{\beta_{p}}, 1, \frac{\beta_{p}}{\alpha_{p}}$.

Let now $\pi=\pi_{f}$ be the automorphic representation attached to the nonCM form $f$ as above, and let $\operatorname{Ad}(\pi)=\operatorname{ad}(\pi) \oplus 1$ be the automorphic form on $\mathrm{GL}_{4}\left(\mathbb{A}_{\mathbb{Q}}\right)$ obtained from the Gelbart-Jacquet adjoint lift by adding the trivial representation. Finally, let

$$
\Pi=\operatorname{Ad}(\pi)(k-1)
$$

be the automorphic representation on $\mathrm{GL}_{4}\left(\mathbb{A}_{\mathbb{Q}}\right)$ obtained by taking the $(k-1)$-st twist of $\operatorname{Ad}(\pi)$.

The following meta-theorem may be considered as a summary of all the results of this paper.

THEOREM 1: If $v$ is a finite place of $F$, then the class of $X_{v}$ in $\operatorname{Br}\left(F_{v}\right)$ is determined by the parity of the slope $m_{v} \in \mathbb{Z} \cup\{\infty\}$ of $\Pi$ at $v$, when this slope is finite. 
Before we proceed further, we wish to remark that the theorem above is another instance of a recurring theme in the theory of the arithmetic of automorphic forms, wherein arithmetic information about an object attached to a form (in this case the endomorphism algebra) is contained in the Fourier coefficients of a suitable lift of the original form (in this case the twisted adjoint lift). The most striking example of this theme occurs in the correspondence between forms of even integral weight $k$ and forms of half-integral weight $(k+1) / 2$ as in [Sh73], [Wa81], [KZ91]. Here, twisted central critical $L$-values of the original form on $\mathrm{PGL}_{2}$ occur as Fourier coefficients of the Shimura-Shintani-Waldspurger lift of this form to the metaplectic group $\widetilde{\mathrm{SL}}_{2}$. The meta-theorem above establishes another instance where this theme is played out.

The slope $m_{v}$ of $\Pi$ at a place $v \mid p$ of $F$ in Theorem 1 is defined to be a suitably normalized $v$-adic valuation of the trace of Frobenius at $p$ in the Galois representation corresponding to $\Pi$. In general, the trace depends on a choice of Frobenius, but is independent of this choice for primes of semistable reduction. Equivalently, on the automorphic side, the slope $m_{v}$ may also be defined as a suitably normalized $v$-adic valuation of the sum of certain parameters coming from the local automorphic representation of $\Pi$ at $p$. Though the shape of the trace of Frobenius, or the shape of the specific parameters, vary in different cases, they can be made completely precise. As a result we obtain various explicit versions of the above meta-theorem which we state now.

For instance, suppose that $v \mid p$ with $p \nmid N$, so that $\pi_{p}$ is an unramified representation. Then the slope $m_{v}$ of $\Pi$ at $v$ is the (normalized) $v$-adic valuation of the sum of the Satake parameters of $\Pi_{p}$. Since $\operatorname{Ad}(\pi)$ has Satake parameters $\frac{\alpha_{p}}{\beta_{p}}, 1, \frac{\beta_{p}}{\alpha_{p}}, 1$, we have

$$
\begin{aligned}
m_{v} & :=\left[F_{v}: \mathbb{Q}_{p}\right] \cdot v\left(\left(\frac{\alpha_{p}}{\beta_{p}}+1+\frac{\beta_{p}}{\alpha_{p}}+1\right) \cdot p^{k-1}\right) \\
& =\left[F_{v}: \mathbb{Q}_{p}\right] \cdot v\left(\frac{\left(\alpha_{p}+\beta_{p}\right)^{2}}{\alpha_{p} \beta_{p}} \cdot p^{k-1}\right) \\
& =\left[F_{v}: \mathbb{Q}_{p}\right] \cdot v\left(a_{p}^{2} \epsilon^{-1}(p)\right) \in \mathbb{Z} \cup\{\infty\},
\end{aligned}
$$

where $v$ is normalized so that $v(p)=1$. We remark that $F$ may be considered as the Hecke field of the adjoint lift $\Pi$, since it is generated by the quantities $a_{p}^{2} \epsilon^{-1}(p)$, for $p \nmid N$. Moreover, the slope $m_{v}$ of $\Pi$ at $v$ is an integer because of the local degree term $\left[F_{v}: \mathbb{Q}_{p}\right]$ (unless of course $a_{p}=0$, in which case $m_{v}$ is infinite). We prove (cf. Theorem 10): 
Theorem 2 (Spherical case): Assume $\operatorname{gcd}(p, N)=1$. Let $v$ be a place of $F$ lying over $p$. Assume $a_{p} \neq 0$. Then $X_{v}$ is a matrix algebra over $F_{v}$ if and only if $m_{v}=\left[F_{v}: \mathbb{Q}_{p}\right] \cdot v\left(a_{p}^{2} \epsilon(p)^{-1}\right) \in \mathbb{Z}$ is even.

The case $k=2$ and $m_{v}=0$ (good, ordinary reduction) is due to Ribet [Ri81]. The general case for odd primes, and for $p=2$ when $F=\mathbb{Q}$, was proved in [BG04] and [GGQ05, Thm. 2.2], under a mild hypothesis. Here we include the case $p=2$ for all $F$, and remove this hypothesis. The proof we give in this paper is much simpler, and was motivated by the recent proof of an analogous theorem for weight one forms [BG11] (this is also where the idea of using the adjoint lift in higher weight germinated).

However, the main point of this article is to treat completely the primes of bad reduction, i.e., the primes $v \mid p$ of $F$ with $p \mid N$. Let $N_{p} \geq 1$ be the exponent of the exact power of $p$ dividing $N$. Let $C$ denote the conductor of $\epsilon$ and let $C_{p} \geq 0$ be the exponent of the exact power of $p$ dividing $C$. Note $N_{p} \geq C_{p}$. Since $p \mid N$, we no longer have the Satake parameters of $\pi_{p}$ at our disposal. However, we can replace these numbers by the corresponding eigenvalues of $\ell$ adic Frobenius in the $\ell$-adic Weil-Deligne representation corresponding to $\pi_{p}$, for $\ell \neq p$, or equivalently by [Sa97], with the eigenvalues of crystalline Frobenius on the filtered $(\varphi, N)$-module attached to $\pi_{p}$ as in [GM09], and can still compute the slope of $\Pi$ at $v$.

For example, in the case that $N_{p}=1$ and $C_{p}=0$, it is well known that $\pi_{p}$ is an unramified twist of the Steinberg representation. In this case, the eigenvalues of $\ell$-adic Frobenius are nothing but $\alpha_{p}=a_{p}$ and $\beta_{p}=p a_{p}$, up to multiplication by the same constant. We thus have

$$
\begin{aligned}
m_{v} & :=\left[F_{v}: \mathbb{Q}_{p}\right] \cdot v\left(\frac{\left(\alpha_{p}+\beta_{p}\right)^{2}}{\alpha_{p} \beta_{p}} \cdot p^{k-1}\right) \\
& =\left[F_{v}: \mathbb{Q}_{p}\right] \cdot(k-2) \in \mathbb{Z} .
\end{aligned}
$$

In Theorem 15 we prove

Theorem 3 (Steinberg case): Suppose $v \mid p$ with $N_{p}=1$ and $C_{p}=0$. Then $X_{v}$ is a matrix algebra over $F_{v}$ if and only if $m_{v}=\left[F_{v}: \mathbb{Q}_{p}\right] \cdot(k-2) \in \mathbb{Z}$ is even.

The proof of Theorem 3 uses the structure of the $\ell$-adic Galois representation attached to $f$ at $p$, for $\ell \neq p$, due to Langlands. The case $k=2$ is due to Ribet 
[Ri81], who in fact showed that the algebra $X$ is trivial in the Brauer group of $F$, using the fact that the corresponding residual abelian variety has toric reduction. Ribet's result was extended to forms of even weight $k$ in [BG04, Thm. 1.0.6]. In this paper examples were also given of forms of odd weight for which the endomorphism algebra is ramified at Steinberg primes. The above theorem gives a complete criterion for the ramification of $X$ at Steinberg primes in all weights $k$.

We now turn to the very interesting case when $N_{p}=C_{p} \geq 1$ and $\pi_{p}$ is in the ramified principal series. The behaviour of the local Brauer class in this case is mysterious, but has now become possible to treat using the adjoint lift. The eigenvalues of $\ell$-adic or crystalline Frobenius are not well-defined in this case since the Weil-Deligne parameter corresponding to $\pi_{p}$ is ramified. However, one more or less canonical choice is $\alpha_{p}=a_{p}$ and $\beta_{p}=\bar{a}_{p} \epsilon^{\prime}(p)$, where we decompose $\epsilon=\epsilon^{\prime} \cdot \epsilon_{p}$ into its prime-to- $p$ conductor and $p$-power conductor parts. We then have

$$
\begin{aligned}
m_{v} & :=\left[F_{v}: \mathbb{Q}_{p}\right] \cdot v\left(\frac{\left(\alpha_{p}+\beta_{p}\right)^{2}}{\alpha_{p} \beta_{p}} \cdot p^{k-1}\right) \\
& =\left[F_{v}: \mathbb{Q}_{p}\right] \cdot v\left(a_{p}^{2} \epsilon^{\prime}(p)^{-1}+2 p^{(k-1)}+\bar{a}_{p}^{2} \epsilon^{\prime}(p)\right) \in \mathbb{Z} \cup\{\infty\} .
\end{aligned}
$$

It can be checked that the three-term expression in the last line above is indeed an element of $F$. It is clearly fixed by complex conjugation; it is in fact fixed by all elements of $\Gamma$ (cf. Lemma 16). Note again that $m_{v} \in \mathbb{Z}$ (unless it is infinite). In view of the two previous theorems, one might conjecture:

$$
\text { If } m_{v}<\infty \text {, then } X_{v} \text { is a matrix algebra over } F_{v}
$$

if and only if $m_{v}$ is even.

We prove that (??) is essentially true. In particular, when the slopes of $\alpha_{p}$ and $\beta_{p}$ are unequal, or equivalently, when $m_{v}<\left[F_{v}: \mathbb{Q}_{p}\right] \cdot(k-1)$, we prove (in Theorem 22, for odd primes $p$, and in Theorem 27, for $p=2$ )

Theorem 4 (Ramified principal series unequal slope case): Assume that $v \mid p$ and $N_{p}=C_{p} \geq 1$. Suppose $m_{v}<\left[F_{v}: \mathbb{Q}_{p}\right] \cdot(k-1)$. Then $X_{v}$ is a matrix algebra over $F_{v}$ if and only if

$$
m_{v}=\left[F_{v}: \mathbb{Q}_{p}\right] \cdot v\left(\frac{a_{p}^{2}}{\epsilon^{\prime}(p)}+2 p^{(k-1)}+\frac{\bar{a}_{p}^{2}}{\bar{\epsilon}^{\prime}(p)}\right) \in \mathbb{Z}
$$

is even. 
We remark that while a partial result in the 'if' direction was proved in [GGQ05, Thm. 5.1], Theorem 4 gives complete information about the ramification of $X_{v}$ in the unequal slope case.

When the slopes of $\alpha_{p}$ and $\beta_{p}$ are the same, or equivalently, $m_{v} \geq$ $\left[F_{v}: \mathbb{Q}_{p}\right] \cdot(k-1)$, the guess $(? ?)$ is, somewhat surprisingly, false, even when $m_{v}<\infty$. Counterexamples are given in Examples 5-7 at the end of the paper. This is related to the fact that the eigenvalues of $\ell$-adic Frobenius are not welldefined. To salvage the situation, we introduce two new quantities $m_{v}^{ \pm}$, which may be thought of as replacements of $m_{v}$. Let $e_{v}$ and $f_{v}$ be the ramification index and residue degree of $v \mid p$, let $G_{v}$ be the decomposition group of $F$ at $v$, and set

$$
m_{v}^{ \pm}=e_{v} \cdot v\left(\left(a_{p}^{2} \epsilon^{\prime}(p)^{-1}\right)^{f_{v}} \pm 2 p^{(k-1) f_{v}}+\left(\bar{a}_{p}^{2} \epsilon^{\prime}(p)\right)^{f_{v}}\right) \in \mathbb{Z} \cup\{\infty\},
$$

where $v$ is normalized so the $v(p)=1$. Again the three-term expression lies in $F$, so $m_{v}^{ \pm}$are well-defined, and at least one of $m_{v}^{ \pm}$is finite. Then we prove (see Theorems 28, 30 and 32 for precise statements)

THEOREM 5 (Ramified principal series equal slope case): Assume that $v \mid p$ and $N_{p}=C_{p} \geq 1$. Suppose $m_{v} \geq\left[F_{v}: \mathbb{Q}_{p}\right] \cdot(k-1)$.

(1) If $p$ is odd and the tame part of $\epsilon_{p}$ is not quadratic on $G_{v}$, then $X_{v}$ is a matrix algebra over $F_{v}$ if and only if one of

$$
m_{v}^{ \pm}=e_{v} \cdot v\left(\left(\frac{a_{p}^{2}}{\epsilon^{\prime}(p)}\right)^{f_{v}} \pm 2 p^{(k-1) f_{v}}+\left(\frac{\bar{a}_{p}^{2}}{\bar{\epsilon}^{\prime}(p)}\right)^{f_{v}}\right) \in \mathbb{Z}
$$

is even.

(2) If $p=2$ and $\epsilon_{2}$ is not quadratic on $G_{v}$, there exists an integer $n_{v} \bmod$ 2 depending only on $\epsilon_{2}$ such that $X_{v}$ is a matrix algebra over $F_{v}$ if and only if one of

$$
m_{v}^{ \pm}+n_{v} \in \mathbb{Z}
$$

is even.

(3) Finally, if $p$ is odd and the tame part of $\epsilon_{p}$ is quadratic on $G_{v}$, or if $p=2$ and $\epsilon_{2}$ is quadratic on $G_{v}$, then there is an integer $n_{v} \bmod 2$ defined in terms of a Hilbert symbol $(t, d)_{v}$, with $t$ depending only on $\epsilon_{p}$ and $d$ on an explicit Fourier coefficient of $f$, such that $X_{v}$ is a matrix algebra over $F_{v}$ if and only if a particular choice of

$$
m_{v}^{ \pm}+n_{v} \in \mathbb{Z}
$$

is even. 
In parts (1) and (2), $m_{v}^{ \pm}$have the same parity if both are finite (and if -1 lies in the image of $\epsilon_{p}$ ). Also, the theorem reduces to the previous theorem when the slopes are unequal. Indeed the quantities $m_{v}^{ \pm}=m_{v}$ coincide in the unequal slope case, since $e_{v} f_{v}=\left[F_{v}: \mathbb{Q}_{p}\right]$, and it turns out that $n_{v}=0$ as well. Thus we may think of $n_{v}$ as an error term to the truth of (??) in the equal slope case.

The above results give a complete answer to Ribet's question on the Brauer class of $X_{f}=X$ in the cases of finite slope. These results cover all forms $f$ of square-free level, and more generally all forms $f$ for which $M_{f}$ has either semistable or crystabelian (crystalline over an abelian extension of $\mathbb{Q}$ ) reduction. The remaining finite places of bad reduction occur when $N_{p}>C_{p}$. In such cases $a_{p}=0$ and even the slope of $f$ is not finite. We hope to return to the infinite slope cases in subsequent work (for a weak result, see Proposition 33).

In closing, we note that although Theorems 2 through 5 are proved separately, there is the tantalizing possibility that there is a more uniform, conceptual proof of these results along the following lines. The Tate conjecture for the motive $M_{f}$ says that the natural map $X_{f} \otimes \mathbb{Q}_{\ell} \rightarrow \operatorname{End}\left(M_{\ell}\right)^{H}$ is an isomorphism, for any prime $\ell$, and for a sufficiently deep finite index subgroup $H$ of the full Galois group $G_{\mathbb{Q}}$ (cf. [Ri80] and [GGQ05]). Here $M_{\ell}$ is the $\ell$-adic realization of $M_{f}$. Now $G_{\mathbb{Q}}$ acts via the adjoint (conjugation) action on $M_{\ell}$, so clearly the local algebra $X \otimes \mathbb{Q}_{\ell}$ and certain fixed points in the adjoint representation of $M_{\ell}$ are related. Moreover, Pink [Pi98] has shown that a compact subgroup of $\mathrm{GL}_{n}$ over a local field is essentially determined by its $\mathfrak{s l}_{n}$-adjoint representation. It would be interesting to see if these remarks can be made into a direct proof of Theorem 1.

\section{Functoriality and the adjoint lift}

We start by recalling a few more details about the adjoint lift mentioned above.

2.1. Functoriality. Let $\mathrm{H}$ and $\mathrm{G}$ be reductive algebraic groups defined over $\mathbb{Q}$, and let ${ }^{L} \mathrm{H}={ }^{L} \mathrm{H}^{0} \rtimes G_{\mathbb{Q}}$ and ${ }^{L} \mathrm{G}={ }^{L} \mathrm{G}^{0} \rtimes G_{\mathbb{Q}}$ be the corresponding $L$-groups. Let $u:{ }^{L} \mathrm{H} \rightarrow{ }^{L} \mathrm{G}$ be an $L$-homomorphism (this map is identity on the second factor). According to Langlands' principle of functoriality there should be a way to lift automorphic forms on $\mathrm{H}\left(\mathbb{A}_{\mathbb{Q}}\right)$ to those on $\mathrm{G}\left(\mathbb{A}_{\mathbb{Q}}\right)$, using the map $u$.

The lifting is in fact done locally. Let $G_{p} \subset G_{\mathbb{Q}}$ be the decomposition subgroup at the prime $p$. The corresponding local $L$-groups are ${ }^{L} \mathrm{H}_{p}={ }^{L} \mathrm{H}^{0} \rtimes G_{p} \subset{ }^{L} \mathrm{H}$ 
and ${ }^{L} \mathrm{G}_{p}={ }^{L} \mathrm{G}^{0} \rtimes G_{p} \subset{ }^{L} \mathrm{G}$. Let $u_{p}:{ }^{L} \mathrm{H}_{p} \rightarrow{ }^{L} \mathrm{G}_{p}$ be the local $L$-homomorphism obtained by restricting $u$ on the second factor to $G_{p}$. We now define the local lift with respect to the local $L$-homomorphism $u_{p}$. Let $\pi_{p}$ be an irreducible admissible representation of $\mathrm{H}\left(\mathbb{Q}_{p}\right)$, with parameter an admissible homomorphism $\phi_{p}: W_{p}^{\prime} \rightarrow{ }^{L} \mathrm{H}_{p}$, where $W_{p}^{\prime}$ is the Weil-Deligne group at $p$. The composition $\phi_{p}^{\prime}=u_{p} \circ \phi_{p}$ is an admissible (if $\mathrm{G}$ is quasi-split) homomorphism of $W_{p}^{\prime}$ to ${ }^{L} \mathrm{G}_{p}$. Then $\phi^{\prime}$ (conjecturally) parametrises a local $L$-packet and the elements of this $L$-packet are the local functorial lifts $\Pi_{p}$ of $\pi_{p}$.

Let now $\pi=\otimes^{\prime} \pi_{p}$ be an irreducible automorphic representation of $\mathrm{H}\left(\mathbb{A}_{\mathbb{Q}}\right)$. An automorphic representation $\Pi=\otimes^{\prime} \Pi_{p}$ of $G\left(\mathbb{A}_{\mathbb{Q}}\right)$ is a weak functorial lift of $\pi$ with respect to $u$, if for all but finitely many places $p, \Pi_{p}$ is a local functorial lift of $\pi_{p}$ with respect to $u_{p}$. Similarly, we call $\Pi$ a strong functorial lift of $\pi$, if $\Pi_{p}$ is a local functorial lift of $\pi_{p}$, for all places $p$. By definition, if $\Pi$ is a weak lift of $\pi$, then for all representations $r:{ }^{L} \mathrm{G} \rightarrow \mathrm{GL}_{n}(\mathbb{C})$ we have the identity of partial Langlands $L$-functions

$$
L_{S}(s, \pi, r \circ u)=L_{S}(s, \Pi, r),
$$

where $S$ is a finite set of places where we do not know how to locally lift $\pi_{p}$. Note $S=\emptyset$ if $\Pi$ is a strong functorial lift of $\pi$.

2.2. Adjoint Lift. Now suppose that $\mathrm{H}=\mathrm{GL}_{2}$ and $\mathrm{G}=\mathrm{GL}_{3}$ are defined over $\mathbb{Q}$. By definition, the connected parts of the corresponding $L$-groups are ${ }^{L} \mathrm{H}^{0}=\mathrm{GL}_{2}(\mathbb{C})$ and ${ }^{L} \mathrm{G}^{0}=\mathrm{GL}_{3}(\mathbb{C})$. The adjoint action of $\mathrm{GL}_{2}(\mathbb{C})$ on the Lie algebra of $\mathrm{SL}_{2}(\mathbb{C})$, namely the trace zero matrices of $\mathrm{M}_{2 \times 2}(\mathbb{C})$, induces $L$ homomorphisms $u$, and $u_{p}$, for each prime $p$. On diagonal elements (of the first factor) the map $u_{p}$ is easily checked to be given by

$$
\left(\begin{array}{cc}
\alpha & 0 \\
0 & \beta
\end{array}\right) \mapsto\left(\begin{array}{ccc}
\frac{\alpha}{\beta} & 0 & 0 \\
0 & 1 & 0 \\
0 & 0 & \frac{\beta}{\alpha}
\end{array}\right) .
$$

By a classical theorem of Gelbart and Jacquet [GJ78], every automorphic representation of $\mathrm{H}$ has a strong lift to G. If $\pi=\pi_{f}$ is the automorphic representation of $\mathrm{GL}_{2}\left(\mathbb{A}_{\mathbb{Q}}\right)$ corresponding to $f \in S_{k}(N, \epsilon)$, let ad $(\pi)$ denote the automorphic lift to $G\left(\mathbb{A}_{\mathbb{Q}}\right)$. The image of arithmetic Frobenius Frob $p$ at $p$ under $\phi_{p}$ is of the form

$$
\left(\left(\begin{array}{cc}
\alpha_{p} & 0 \\
0 & \beta_{p}
\end{array}\right), \operatorname{Frob}_{p}\right) .
$$


If $p \nmid N$ is an unramified prime, $\alpha_{p}$ and $\beta_{p}$ are the Satake parameters of $\pi_{p}$. Then by definition of $u_{p}$ it is clear that the image of $\operatorname{Frob}_{p}$ under $\phi_{p}^{\prime}$ is a diagonal matrix with entries $\frac{\alpha_{p}}{\beta_{p}}, 1, \frac{\beta_{p}}{\alpha_{p}}$ (on the first factor, and just Frob ${ }_{p}$ on the second factor).

It is more convenient to work with $\Pi=(\operatorname{ad}(\pi) \oplus 1)(k-1)$, the $(k-1)$-th twist of the automorphic representation on $\mathrm{GL}_{4}\left(\mathbb{A}_{\mathbb{Q}}\right)$ obtained by adding the trivial representation to $\operatorname{ad}(\pi)$. We define the slope $m_{v}$ of $\Pi$ at $v \mid p$ to be

$$
m_{v}:=\left[F_{v} \cdot \mathbb{Q}_{p}\right] \cdot v\left(t_{p}\right),
$$

where $v$ is normalized so that $v(p)=1$ and $t_{p} \in F$ is defined to be the sum of the four parameters of $\Pi_{p}$, namely

$$
t_{p}=\left(\frac{\alpha_{p}}{\beta_{p}}+1+\frac{\beta_{p}}{\alpha_{p}}+1\right) \cdot p^{k-1}=\frac{\left(\alpha_{p}+\beta_{p}\right)^{2}}{\alpha_{p} \beta_{p}} \cdot p^{k-1} .
$$

We note that $t_{p}$ can be computed easily in various cases. When $p \nmid N$ an easy check shows

$$
t_{p}=\frac{a_{p}^{2}}{\epsilon(p)}
$$

When $p \mid N$ and $N_{p}=1$ and $C_{p}=0$, it is known that $\alpha_{p}=a_{p}$ and $\beta_{p}=p a_{p}$ (up to multiplication by a constant), and so

$$
t_{p}=p^{k-2}(p+1)^{2} .
$$

Finally, if $N_{p}=C_{p}$, then a natural choice is $\alpha_{p}=a_{p}$ and $\beta_{p}=\bar{a}_{p} \epsilon^{\prime}(p)$ (again up to multiplication by a constant), so

$$
t_{p}=\frac{a_{p}^{2}}{\epsilon^{\prime}(p)}+2 p^{k-1}+\frac{\bar{a}_{p}^{2}}{\bar{\epsilon}^{\prime}(p)}
$$

noting $\left|a_{p}\right|^{2}=p^{k-1}$. In fact the Weil-Deligne parameter in this case is ramified at $p$, so there are other choices for $\alpha_{p}$ and $\beta_{p}$ and hence for $t_{p}$. This causes some complications in the statements and the proofs of results in this case.

2.3. Galois representations. All the above formulas can be computed on the Galois side as well. Let $\rho_{f}: G_{\mathbb{Q}} \rightarrow \mathrm{GL}_{2}\left(E_{\lambda}\right)$ be an $\ell$-adic Galois representation attached by Deligne to $f$, for a prime $\lambda \mid \ell$ of $E$ with $\ell \neq p$. Let $\lambda(x)$ be the unramified character which takes arithmetic Frobenius Frob $p$ to $x \in E_{\lambda}$.

Theorem 6 (Langlands): The local behaviour of $\left.\rho_{f}\right|_{G_{p}}$ at a decomposition group $G_{p}$ at $p$ is as follows. 
- If $p \nmid N$, let $\alpha_{p}$ and $\beta_{p}$ be roots of the polynomial $x^{2}-a_{p} x+\epsilon(p) p^{k-1}$. Then

$$
\left.\rho_{f}\right|_{G_{p}} \sim\left(\begin{array}{cc}
\lambda\left(\beta_{p}\right) & 0 \\
0 & \lambda\left(\alpha_{p}\right)
\end{array}\right) .
$$

- If $N_{p}=1$ and $C_{p}=0$, let $\alpha_{p}=a_{p}$ and $\beta_{p}=p a_{p}$. Then

$$
\left.\rho_{f}\right|_{G_{p}} \sim\left(\begin{array}{cc}
\lambda\left(\beta_{p}\right) & * \\
0 & \lambda\left(\alpha_{p}\right)
\end{array}\right) .
$$

- If $N_{p}=C_{p} \geq 1$, let $\alpha_{p}=a_{p}$ and $\beta_{p}=\bar{a}_{p} \epsilon^{\prime}(p)$. Then

$$
\left.\rho_{f}\right|_{G_{p}} \sim\left(\begin{array}{cc}
\lambda\left(\beta_{p}\right) \cdot \epsilon_{p} & 0 \\
0 & \lambda\left(\alpha_{p}\right)
\end{array}\right) .
$$

- If $N_{p} \geq 2>C_{p}$ and $p>2$, and $\pi_{p}$ is supercuspidal, then $\left.\rho_{f}\right|_{G_{p}} \sim$ $\operatorname{Ind}_{G_{K}}^{G_{p}} \chi$, for a quadratic extension $K$ of $\mathbb{Q}_{p}$, and a character $\chi$ of $G_{K}$.

Let $\pi=\pi_{f}$ be the automorphic representation corresponding to $f$. Then $\rho_{\pi}$, the Galois representation attached to $\pi$, differs a bit from $\rho_{f}$ (e.g., the Satake parameters differ from the roots of the polynomial $x^{2}-a_{p} x+\epsilon(p) p^{k-1}$ by a factor of $p^{(k-1) / 2}$, and similarly the $L$-functions satisfy $L(s, f)=L\left(s-\frac{k-1}{2}, \pi, 1\right)$ ). However, the resulting adjoint Galois representation obtained by making $G_{\mathbb{Q}}$ act by conjugation on $\mathrm{M}_{2 \times 2}\left(E_{\lambda}\right)$ is the same, and we let

$$
\rho_{\mathrm{Ad}(\pi)}: G_{\mathbb{Q}} \rightarrow \mathrm{GL}_{4}\left(E_{\lambda}\right)
$$

be defined by $\rho_{\operatorname{Ad}(\pi)}(g)(X)=\rho_{\pi}(g) X \rho_{\pi}(g)^{-1}$, for all $X \in \mathrm{M}_{2 \times 2}\left(E_{\lambda}\right)$ and $g \in$ $G_{\mathbb{Q}}$. Finally, let

$$
\rho_{\Pi}=\rho_{\operatorname{Ad}(\pi)} \otimes \chi_{\ell}^{k-1}
$$

be the representation obtained by taking the $(k-1)$-fold twist of the adjoint representation by the $\ell$-adic cyclotomic character.

Corollary 7: We have:

- If $p \nmid N$, then $\operatorname{trace}\left(\rho_{\Pi}\left(\operatorname{Frob}_{p}\right)\right)=a_{p}^{2} / \epsilon(p)$.

- If $N_{p}=1$ and $C_{p}=0$, then $\operatorname{trace}\left(\rho_{\Pi}\left(\operatorname{Frob}_{p}\right)\right)=p^{k-2}(p+1)^{2}$.

- If $N_{p}=C_{p} \geq 1$, then in many cases there exists an arithmetic Frobenius $\operatorname{Frob}_{p}$ such that $\operatorname{trace}\left(\rho_{\Pi}\left(\operatorname{Frob}_{p}\right)\right)=a_{p}^{2} / \epsilon^{\prime}(p)+2 p^{k-1}+\bar{a}_{p}^{2} / \bar{\epsilon}^{\prime}(p)$.

Proof. If

$$
\rho_{\pi}\left(\operatorname{Frob}_{p}\right) \sim\left(\begin{array}{cc}
\alpha_{p} & 0 \\
0 & \beta_{p}
\end{array}\right),
$$


then

$$
\rho_{\operatorname{Ad}(\pi)}\left(\operatorname{Frob}_{p}\right) \sim\left(\begin{array}{cccc}
\frac{\alpha_{p}}{\beta_{p}} & 0 & 0 & 0 \\
0 & 1 & 0 & 0 \\
0 & 0 & \frac{\beta_{p}}{\alpha_{p}} & 0 \\
0 & 0 & 0 & 1
\end{array}\right)
$$

and $\chi_{\ell}\left(\operatorname{Frob}_{p}\right)^{k-1}=p^{k-1}$. Taking the trace of $\rho_{\Pi}\left(\operatorname{Frob}_{p}\right)$ gives the corollary.

\section{The Brauer class of $X$}

3.1. Definition of $\alpha$. Recall that for $\gamma \in \Gamma$, there is a unique $E$-valued Dirichlet character $\chi_{\gamma}$ such that $f^{\gamma}=f \otimes \chi_{\gamma}$, and hence $\rho_{f^{\gamma}} \sim \rho_{f} \otimes \chi_{\gamma}$. For $\gamma, \delta \in \Gamma$, the identity

$$
\chi_{\gamma \delta}=\chi_{\gamma} \chi_{\delta}^{\gamma}
$$

shows that $\gamma \mapsto \chi_{\gamma}$ is a 1-cocycle. Specialising to $g \in G_{\mathbb{Q}}$, we see that $\gamma \mapsto \chi_{\gamma}(g)$ is an $E$-valued 1-cocycle as well. By Hilbert's theorem $90, \mathrm{H}^{1}\left(\Gamma, E^{*}\right)$ is trivial, so there is an element $\alpha(g) \in E^{*}$ such that

$$
\alpha(g)^{\gamma-1}=\chi_{\gamma}(g)
$$

for all $\gamma \in \Gamma$ (cf. [Ri85]). Clearly, $\alpha(g)$ is completely determined up to multiplication by elements of $F^{*}$. Varying $g \in G_{\mathbb{Q}}$, we obtain a well-defined map

$$
\tilde{\alpha}: G_{\mathbb{Q}} \rightarrow E^{*} / F^{*} .
$$

Since each $\chi_{\gamma}$ is a character, $\tilde{\alpha}$ is a homomorphism.

We can and do lift $\tilde{\alpha}$ to a map $\alpha: G_{\mathbb{Q}} \rightarrow E^{*}$. The following result summarizes some well-known properties of these maps. The proofs given in [Ri75, Thm. 1.1], [Ri04, Thm. 5.5] for $k=2$ (see also [Ri85], [BG11, Lem. 9]) easily extend to higher weight.

Proposition 8 (Ribet): We have:

(1) $\tilde{\alpha}: G_{\mathbb{Q}} \rightarrow E^{*} / F^{*}$ is unramified at all primes $p$ of semistable reduction.

(2) $\alpha^{2}(g) \equiv \epsilon(g) \bmod F^{*}$, for all $g \in G_{\mathbb{Q}}$.

(3) $\alpha\left(\right.$ Frob $\left._{p}\right) \equiv a_{p} \bmod F^{*}$, for primes $p \nmid N$, if $a_{p} \neq 0$.

(4) $\alpha(g) \equiv \operatorname{trace}\left(\rho_{f}(g)\right) \bmod F^{*}$, for $g \in G_{\mathbb{Q}}$, if $\operatorname{trace}\left(\rho_{f}(g)\right) \neq 0$. 
3.2. The 2-COCYCle $c_{\alpha}$. By [Ri81, Prop. 1], whose proof holds for weights $k \geq 2$ as well, the class of $X$ in $\operatorname{Br}(F)=\mathrm{H}^{2}\left(G_{F}, \overline{\mathbb{Q}}^{*}\right)$ is given by the 2-cocycle $(g, h) \mapsto \chi_{g}(h)$, for $g, h \in G_{F}$, where $\chi_{g}:=\chi_{\gamma}$ for $\gamma$ the image of $g$ in $\Gamma$. By the definition of $\alpha$, this 2-cocycle is the same as the 2-cocycle given by $(g, h) \mapsto \frac{\alpha(h)^{g}}{\alpha(h)}$, which differs from the 2-cocycle

$$
c_{\alpha}(g, h)=\frac{\alpha(g) \alpha(h)}{\alpha(g h)}
$$

by a coboundary. Hence, the class of $X$ is given by the 2 -cocycle $c_{\alpha}(g, h)$ above.

Observe that the class of $c_{\alpha}$ is independent of the lift $\alpha$ of $\tilde{\alpha}$. Suppose $\alpha^{\prime}$ is another lift of $\tilde{\alpha}$. Then $\alpha^{\prime}(g)=\alpha(g) f(g)$, for some map $f: G_{F} \rightarrow F^{*}$. Then $c_{\alpha}$ and $c_{\alpha^{\prime}}$ differ by the map $(g, h) \mapsto \frac{f(g) f(h)}{f(g h)}$, which is clearly a 2-coboundary.

We also note that the class of $c_{\alpha}$ (hence $X$ ) is 2-torsion in the Brauer group of $F$, since $c_{\alpha}^{2}(g, h)=\frac{d(g) d(h)}{d(g h)}$ is a 2-coboundary, where $d(g):=\alpha^{2}(g) / \epsilon(g) \in F^{*}$, by part (2) of Proposition 8.

3.3. Invariant map. To study the Brauer class of $X$, it suffices to study the Brauer class of $X_{v}:=X \otimes_{F} F_{v}$ in $\operatorname{Br}\left(F_{v}\right)$, for each place $v$ of $F$. It is well known that if $v$ is finite, then

$$
\operatorname{inv}_{v}: \operatorname{Br}\left(F_{v}\right) \simeq \mathbb{Q} / \mathbb{Z}
$$

via the invariant map $\operatorname{inv}_{v}$ at $v$. Since the class of $X$ is 2-torsion in the Brauer group of $F$, we have that $\operatorname{inv}_{v}\left(X_{v}\right) \in \frac{1}{2} \mathbb{Z} / \mathbb{Z}$. Identifying this group with $\mathbb{Z} / 2$, we see that $X_{v}$ is a matrix algebra over $F_{v}$ if $\operatorname{inv}_{v}\left(X_{v}\right)=0 \bmod 2$, and is a matrix algebra over a quaternion division algebra over $F_{v}$ if $\operatorname{inv}_{v}\left(X_{v}\right)=1 \bmod$ 2 .

To aid in the computation of $\operatorname{inv}_{v}\left(X_{v}\right)$, for finite places $v$, it is useful to recall the explicit definition of the invariant map, which we do now. Let $I_{v}$ be the inertia subgroup of $G_{F}$ at the prime $v$. Let $\operatorname{Gal}\left(F_{v}^{\mathrm{nr}} / F_{v}\right)$ be the Galois group of $F_{v}^{\mathrm{nr}}$, the maximal unramified extension of $F_{v}$, over $F_{v}$. The inflation map

$$
\operatorname{Inf}: \mathrm{H}^{2}\left(\operatorname{Gal}\left(F_{v}^{\mathrm{nr}} / F_{v}\right), F_{v}^{\mathrm{nr}}\right) \rightarrow \operatorname{Br}\left(F_{v}\right)
$$

is well-known to be an isomorphism. Now, the surjective valuation $v: F^{*} \rightarrow \mathbb{Z}$ can be extended uniquely to $\left(F_{v}^{\mathrm{nr}}\right)^{*}$ which we continue to call $v$. This gives rise to a map

$$
v: \mathrm{H}^{2}\left(\operatorname{Gal}\left(F_{v}^{\mathrm{nr}} / F_{v}\right), F_{v}^{\mathrm{nr}}\right) \rightarrow \mathrm{H}^{2}\left(\operatorname{Gal}\left(F_{v}^{\mathrm{nr}} / F_{v}\right), \mathbb{Z}\right)
$$


which we again denote by $v$. Also, the short exact sequence of abelian groups

$$
0 \rightarrow \mathbb{Z} \rightarrow \mathbb{Q} \rightarrow \mathbb{Q} / \mathbb{Z} \rightarrow 0
$$

gives rise to a long exact sequence of cohomology groups, with boundary map

$$
\delta: \mathrm{H}^{1}\left(\operatorname{Gal}\left(F_{v}^{\mathrm{nr}} / F_{v}\right), \mathbb{Q} / \mathbb{Z}\right) \rightarrow \mathrm{H}^{2}\left(\operatorname{Gal}\left(F_{v}^{\mathrm{nr}} / F_{v}\right), \mathbb{Z}\right)
$$

which is an isomorphism since $\mathrm{H}^{i}\left(\operatorname{Gal}\left(F_{v}^{\mathrm{nr}} / F_{v}\right), \mathbb{Q}\right)=0$ for $i=1,2$. We recall the definition of $\delta$. If $\chi: \operatorname{Gal}\left(F_{v}^{\mathrm{nr}} / F_{v}\right) \rightarrow \mathbb{Q} / \mathbb{Z}$ is a homomorphism, and $\tilde{\chi}$ is a lift of $\chi$ to $\mathbb{Q}$, then $\delta(\chi)$ is the $\mathbb{Z}$-valued 2-cocycle on $\operatorname{Gal}\left(F_{v}^{\mathrm{nr}} / F_{v}\right)$ given by

$$
(g, h) \mapsto \frac{\tilde{\chi}(g) \tilde{\chi}(h)}{\tilde{\chi}(g h)} .
$$

Finally, there is a map, say Ev (for evaluation)

$$
\mathrm{Ev}: \mathrm{H}^{1}\left(\operatorname{Gal}\left(F_{v}^{\mathrm{nr}} / F_{v}\right), \mathbb{Q} / \mathbb{Z}\right) \rightarrow \mathbb{Q} / \mathbb{Z},
$$

obtained by evaluating a homomorphism at the arithmetic Frobenius at $v$. Then, by definition, the invariant map at $v$ is given by

$$
\operatorname{inv}_{v}=\operatorname{Ev} \circ \delta^{-1} \circ v \cdot \operatorname{Inf}^{-1}: \operatorname{Br}\left(F_{v}\right) \rightarrow \mathbb{Q} / \mathbb{Z} .
$$

3.4. Local 2-COCycle. Now let $K: G_{v} \rightarrow \bar{F}_{v}^{*}$ be any map. Then

$$
c_{K}(g, h)=\frac{K(g) K(h)}{K(g h)}
$$

defines a local 2-cocycle on $G_{v}$, if $c_{K}(g, h) \in F_{v}$, for all $g, h \in G_{v}$. We call it the local 2-cocycle defined by the function $K$. The following general lemma regarding the Brauer class of this local 2-cocycle will be very useful in computations.

Lemma 9: Let $K: G_{v} \rightarrow \bar{F}_{v}^{*}$ be a map and let $t: G_{v} \rightarrow \bar{F}_{v}^{*}$ be an unramified homomorphism such that

(1) $K(i) \in F_{v}^{*}$, for all $i \in I_{v}$,

(2) $K(g)^{2} / t(g) \in F_{v}^{*}$, for all $g \in G_{v}$.

Then, for any arithmetic Frobenius Frob $_{v}$ at $v$, we have

$$
\operatorname{inv}_{v}\left(c_{K}\right)=\frac{1}{2} \cdot v\left(\frac{K\left(\operatorname{Frob}_{v}\right)^{2}}{t\left(\operatorname{Frob}_{v}\right)}\right) \in \frac{1}{2} \mathbb{Z} / \mathbb{Z},
$$

where $v: F_{v}^{*} \rightarrow \mathbb{Z}$ is the surjective valuation. 
Proof. We will calculate $\operatorname{inv}_{v}\left(c_{K}\right)$, step by step, using the definition of $\operatorname{inv}_{v}$ just recalled.

Replacing the induced homomorphism $K: G_{v} \rightarrow \bar{F}_{v}^{*} / F_{v}^{*}$ with another lift $K: G_{v} \rightarrow \bar{F}_{v}^{*}$ which we again call $K$ does not change the cohomology class of $c_{K}$. By property (1) we may choose a lift $K$ such that for $g \in G_{v}, K(g i)=K(g)$, for all $i \in I_{v}$. Denote the image of $g$ under the projection map $G_{v} \rightarrow G_{v} / I_{v}=\hat{\mathbb{Z}}$ by $\bar{g}$. Define $c_{\bar{K}}: \hat{\mathbb{Z}} \times \hat{\mathbb{Z}} \rightarrow F_{v}^{*}$ by $c_{\bar{K}}(\bar{g}, \bar{h})=c_{K}(g, h)$. Then $c_{\bar{K}}$ is clearly a well-defined 2-cocycle on $\hat{\mathbb{Z}}$ whose image under the inflation map is $c_{K}$.

Now, by definition, $v\left(c_{\bar{K}}\right)$ is the 2-cocycle defined by

$$
(g, h) \mapsto v\left(\frac{K(g) K(h)}{K(g h)}\right) \in \mathbb{Z},
$$

for $g, h \in G_{v}$.

By property (2), $d(g)=K^{2}(g) / t(g) \in F_{v}^{*}$, for $g \in G_{v}$. The 2-cocycle above is the same as the 2 -cocycle induced by

$$
(g, h) \mapsto \frac{1}{2} \cdot v\left(\frac{d(g) d(h)}{d(g h)}\right) \in \mathbb{Z} .
$$

Consider now the map $\chi: \operatorname{Gal}\left(F_{v}^{\mathrm{nr}} / F_{v}\right) \rightarrow \mathbb{Q} / \mathbb{Z}$ defined by

$$
\chi(g)=\frac{1}{2} \cdot v(d(g)) \bmod \mathbb{Z}
$$

Under the boundary map $\delta$ the 1-cocycle $\chi$ maps to the 2-cocycle above, so $\left.\left(\delta^{-1} \circ v \circ \operatorname{Inf}^{-1}\right)\left(c_{K}\right)\right)$ is just $\chi$. Hence

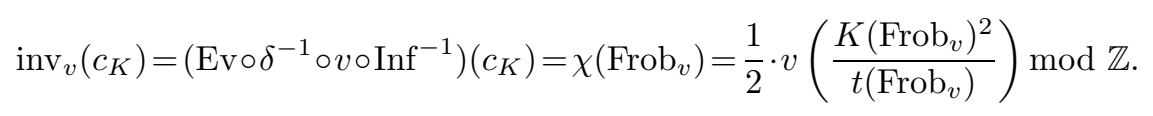

\section{Good primes}

Theorem 10: Assume $\operatorname{gcd}(p, N)=1$ and assume $a_{p} \neq 0$. Let $v$ be a place of $F$ lying over $p$. Then $X_{v}$ is a matrix algebra over $F_{v}$, if and only if the slope

$$
m_{v}=\left[F_{v}: \mathbb{Q}_{p}\right] \cdot v\left(a_{p}^{2} / \epsilon(p)\right) \in \mathbb{Z}
$$

is even, where $v$ is normalized such that $v(p)=1$.

Proof. This follows immediately from the lemma above taking $K=\alpha$ and $t=\epsilon$. Indeed, we have $\operatorname{inv}_{v}\left(c_{\alpha}\right)=\frac{1}{2} v\left(\alpha^{2}\left(\operatorname{Frob}_{v}\right) / \epsilon\left(\operatorname{Frob}_{v}\right)\right) \bmod \mathbb{Z}$, and $\alpha\left(\operatorname{Frob}_{v}\right) \equiv$ $a_{p}^{f_{v}} \bmod F^{*}$, by part (3) of Proposition 8. 
For the cases where $a_{p}=0$ we have the following criterion (which is not in terms of a slope). Let $p^{\dagger} \nmid N$ be a prime such that $p^{\dagger} \equiv p \bmod N$ and $a_{p^{\dagger}} \neq 0$. Let

$$
m_{v}^{\dagger}:=\left[F_{v}: \mathbb{Q}_{p^{\dagger}}\right] \cdot v\left(a_{p^{\dagger}}^{2} / \epsilon\left(p^{\dagger}\right)\right) \in \mathbb{Z}
$$

where $v$ is normalized so that $v(p)=1$.

Theorem 11: Let $\operatorname{gcd}(p, N)=1$ and suppose $a_{p}=0$. Let $v$ be a place of $F$ lying over $p$. Then $X_{v}$ is a matrix algebra over $F_{v}$ if and only if $m_{v}^{\dagger} \in \mathbb{Z}$ is even.

Proof. The proof is similar to that of the previous theorem, with minor changes. Note that $p^{\dagger} \equiv p \bmod N$ implies $\chi_{\gamma}(p)=\chi_{\gamma}\left(p^{\dagger}\right)$, for all $\gamma \in \Gamma$. So, if Frob $p$ and Frob $_{p^{\dagger}}$ denote the Frobenii at the prime $p$ and $p^{\dagger}$, then by (3.1), we have $\alpha\left(\operatorname{Frob}_{p}\right) \equiv \alpha\left(\right.$ Frob $\left._{p^{\dagger}}\right) \equiv a_{p^{\dagger}} \bmod F^{*}$. Hence

$$
\begin{aligned}
\operatorname{inv}_{v}\left(c_{\alpha}\right) & =\frac{1}{2} v\left(\frac{\alpha^{2}\left(\operatorname{Frob}_{v}\right)}{\epsilon\left(\operatorname{Frob}_{v}\right)}\right)=\frac{1}{2} \cdot f_{v} \cdot v\left(\frac{\alpha^{2}\left(\operatorname{Frob}_{p}\right)}{\epsilon(p)}\right) \\
& =\frac{1}{2} \cdot f_{v} \cdot v\left(\frac{a_{p^{\dagger}}^{2}}{\epsilon\left(p^{\dagger}\right)}\right) \bmod \mathbb{Z} .
\end{aligned}
$$

\section{Steinberg primes}

Let us now turn to the cases where $p \mid N$. In this section we assume that $N_{p}=1$ and $C_{p}=0$. Thus $N=M p$, where $M$ is a positive integer with $(M, p)=1$, and $\epsilon$ is a character $\bmod M$.

Lemma 12: If $\left(\gamma, \chi_{\gamma}\right)$ is an extra twist for $f$, then the conductor of $\chi_{\gamma}$ divides M.

Proof. A general result due to Atkin-Li [AL78, Thm. 3.1] allows one to calculate the exact level of the newform attached to a twisted form $f \otimes \chi$. We recall this now. Let $f \in S_{k}(N, \epsilon)$ be a newform of weight $k \geq 2$, and nebentypus $\epsilon$. In the notation of loc. cit., let $q \mid N$ be a prime and let $Q$ be the $q$-primary factor of $N$. So $N=Q M$, with $(M, q)=1$. Let the conductor of $\epsilon_{Q}$, the $q$-part of $\epsilon$, be $q^{\alpha}$, for $\alpha \geq 0$. Let $\chi$ be a character of conductor $q^{\beta}$, with $\beta \geq 1$. Set

$$
Q^{\prime}=\operatorname{Max}\left\{Q, q^{\alpha+\beta}, q^{2 \beta}\right\} .
$$

According to the theorem, the level of the newform attached to $f \otimes \chi_{\gamma}$ is $Q^{\prime} M$, provided that 
- $\max \left\{q^{\alpha+\beta}, q^{2 \beta}\right\} \leq Q$, if $Q^{\prime}=Q$, or

- Conductor of $\epsilon_{Q \chi}=\max \left\{q^{\alpha}, q^{\beta}\right\}$, if $Q^{\prime}>Q$.

In our case, taking $Q=q=p$, we have $\epsilon_{Q}=\epsilon_{p}=1$. We let $\chi$ be the $p$-part of $\chi_{\gamma}$. Suppose towards a contradiction that $\chi_{\gamma}$ has level divisible by $p$. Then $\alpha=0$ and $\beta=1$. Then $Q^{\prime}=p^{2}>Q=p$ and the $Q$-part of the conductor of $\epsilon_{Q} \chi_{\gamma}=\chi_{\gamma}$ is $p$. So the second condition above is satisfied and we get the $p$-part of the level of the newform attached to $f \otimes \chi_{\gamma}$ is $p^{2}$. On the other hand, $f \otimes \chi_{\gamma}=f^{\gamma}$ has the same level as $f$, namely $M p$, which is not divisible by $p^{2}$, a contradiction. Thus the $p$-part of the conductor of $\chi_{\gamma}$ must be trivial, as desired.

Recall that $a_{\ell}^{\gamma}=a_{\ell} \cdot \chi_{\gamma}(\ell)$ for all primes $\ell \nmid N$. We show that this also holds for $p \| N$.

Lemma 13: $a_{p}^{\gamma}=\chi_{\gamma}(p) \cdot a_{p}$, for all $\gamma \in \Gamma$.

Proof. We use the precise form of the local Galois representation at $p$ from Langlands' theorem (Theorem 6, see also [Hi00, Thm. 3.26]). We have

$$
\left.\rho_{f}\right|_{G_{p}} \sim\left(\begin{array}{cc}
\lambda\left(p a_{p}\right) & * \\
0 & \lambda\left(a_{p}\right)
\end{array}\right),
$$

where $\lambda(x): G_{p} \longrightarrow \mathbb{Z}_{l}^{*}$ is the unramified character taking arithmetic Frobenius to $x$. Note that both characters make sense since both $p a_{p}$ and $a_{p}$ are $\ell$ adic units. By the previous lemma, the conductor of $\chi_{\gamma}$, for $\gamma \in \Gamma$, is prime to $p$ and so $\chi_{\gamma}(p)$ makes sense, and is an $\ell$-adic unit, and locally we have $\left.\chi_{\gamma}\right|_{G_{p}}=\lambda\left(\chi_{\gamma}(p)\right)$. Applying Langlands' theorem for $f^{\gamma}$, we get

$$
\left.\rho_{f^{\gamma}}\right|_{G_{p}} \sim\left(\begin{array}{cc}
\lambda\left(p a_{p}^{\gamma}\right) & * \\
0 & \lambda\left(a_{p}^{\gamma}\right)
\end{array}\right) .
$$

Since $f^{\gamma}=f \otimes \chi_{\gamma}$ implies $\rho_{f^{\gamma}} \sim \rho_{f} \otimes \chi_{\gamma}$, we have locally that

$$
\left(\begin{array}{cc}
\lambda\left(p a_{p}^{\gamma}\right) & * \\
0 & \lambda\left(a_{p}^{\gamma}\right)
\end{array}\right) \sim\left(\begin{array}{cc}
\lambda\left(p a_{p}\right) \lambda\left(\chi_{\gamma}(p)\right) & * \\
0 & \lambda\left(a_{p}\right) \lambda\left(\chi_{\gamma}(p)\right)
\end{array}\right) .
$$

An important part of Langlands' theorem (not mentioned explicitly above) is that $* \neq 0$, since the inertia group $I_{p}$ acts unipotently with infinite image. Thus comparing like diagonal entries, we see that $a_{p}^{\gamma}=\chi_{\gamma}(p) \cdot a_{p}$. 
Recall that the map $\tilde{\alpha}: G_{\mathbb{Q}} \rightarrow E^{*} / F^{*}$ is unramified at primes of semistable reduction, and $\alpha\left(\operatorname{Frob}_{p}\right) \equiv a_{p} \bmod F^{*}$ at primes of good reduction (cf. Proposition 8 ). We now observe that this last formula continues to hold for primes of semistable reduction.

Proposition 14: Suppose $p$ is a prime such that $N_{p}=1$ and $C_{p}=0$. Then $\alpha\left(\operatorname{Frob}_{p}\right) \equiv a_{p} \bmod F^{*}$.

Proof. Since, for $\gamma \in \Gamma$, the conductor of $\chi_{\gamma}$ is prime to $p$, we have $\chi_{\gamma}(i)=1$, for $i \in I_{p}$. By (3.1), we deduce $\alpha(i) \in F^{*}$, for all $i \in I_{p}$. Thus we recover the fact that $\tilde{\alpha}$ is unramified at the Steinberg primes for any $k \geq 2$. In any case, it makes sense to speak of $\alpha\left(\operatorname{Frob}_{p}\right) \bmod F^{*}$. By Lemma 13, we have $a_{p}^{\gamma-1}=\chi_{\gamma}(p)$, for $\gamma \in \Gamma$. By $(3.1), \alpha\left(\operatorname{Frob}_{p}\right)^{\gamma-1}=\chi_{\gamma}(p)$. Since these identities hold for all $\gamma \in \Gamma$, we deduce that $\alpha\left(\operatorname{Frob}_{p}\right) \equiv a_{p} \bmod F^{*}$.

Theorem 15: Let $N_{p}=1$ and $C_{p}=0$ and let $v \mid p$ be a prime of $F$. Then $X_{v}$ is a matrix algebra if and only if $\left[F_{v}: \mathbb{Q}_{p}\right] \cdot(k-2)$ is even.

Proof. Applying Lemma 9 to $K=\alpha$ and $t=\epsilon$, we get

$$
\operatorname{inv}_{v}\left(c_{\alpha}\right)=\frac{1}{2} v\left(\frac{\alpha^{2}\left(\operatorname{Frob}_{v}\right)}{\epsilon\left(\operatorname{Frob}_{v}\right)}\right) \bmod \mathbb{Z} .
$$

By the previous proposition, $\alpha\left(\operatorname{Frob}_{v}\right)=a_{p}^{f_{v}} \bmod F^{*}$. Thus

$$
\operatorname{inv}_{v}\left(c_{\alpha}\right)=\frac{1}{2} \cdot f_{v} \cdot v\left(\frac{a_{p}^{2}}{\epsilon(p)}\right)
$$

By Theorem 4.6.17 [Mi89],

$$
\frac{a_{p}^{2}}{\epsilon_{M}(p)}=p^{k-2}
$$

Also, we may replace the valuation $v$ by $e_{v} \cdot v$, where the second $v$ is normalized such that $v(p)=1$. We obtain that $\operatorname{inv}_{v}\left(c_{\alpha}\right)=\left[F_{v}: \mathbb{Q}_{p}\right] \cdot(k-2) \bmod 2$, as desired.

\section{Ramified principal series primes}

We now assume that $N_{p}=C_{p} \geq 1$. Let $v$ be a place of $F$ lying above $p$. Let $e_{v}$ and $f_{v}$ be the ramification degree and inertia degree of $v$ over $p$. Recall that in this case $\pi_{p}$ is in the ramified principal series. 
Recall that $\epsilon=\epsilon^{\prime} \cdot \epsilon_{p}$ is a decomposition of the nebentypus $\epsilon$ into its primeto- $p$ part and $p$ part. We use repeatedly a fundamental theorem of Langlands (Theorem 6), which states that the local Galois representation at the prime $p$ is given by

$$
\left.\rho_{f}\right|_{G_{p}} \sim\left(\begin{array}{cc}
\lambda\left(\bar{a}_{p} \epsilon^{\prime}(p)\right) \cdot \epsilon_{p} & 0 \\
0 & \lambda\left(a_{p}\right)
\end{array}\right),
$$

where $\lambda(x)$ is the usual local unramified character.

LEMma 16: Let $\mu=\frac{a_{p}^{2}}{\epsilon^{\prime}(p)}$ and $\nu=\bar{\mu}=\frac{\bar{a}_{p}^{2}}{\bar{\epsilon}^{\prime}(p)}$. Then $\mu^{f}+\nu^{f} \in F$, for all integers $f \geq 1$.

Proof. Let $\left(\gamma, \chi_{\gamma}\right)$ be an extra twist for the form $f$. Thus we have $\rho_{f^{\gamma}} \sim \rho_{f} \otimes \chi_{\gamma}$. Hence, by Langlands' theorem, locally on $G_{p}$ we have

$$
\left(\begin{array}{cc}
\lambda\left(\bar{a}_{p}^{\gamma} \epsilon^{\prime}(p)^{\gamma}\right) \cdot \epsilon_{p}^{\gamma} & 0 \\
0 & \lambda\left(a_{p}^{\gamma}\right)
\end{array}\right) \sim\left(\begin{array}{cc}
\lambda\left(\bar{a}_{p} \epsilon^{\prime}(p)\right) \cdot \epsilon_{p} \cdot \chi_{\gamma} & 0 \\
0 & \lambda\left(a_{p}\right) \cdot \chi_{\gamma}
\end{array}\right) .
$$

One of the two characters on the left is unramified and the other one is ramified. Thus the same must be true on the right-hand side. Moreover, the unramified characters on both sides must be equal and the ramified characters must also be equal.

We decompose $\chi_{\gamma}$ into its prime-to- $p$ and $p$ parts, namely $\chi_{\gamma}=\chi_{\gamma}^{\prime} \cdot \chi_{\gamma, p}$. First, assume that $\chi_{\gamma}$ is unramified at $p$. Then, $\chi_{\gamma}=\chi_{\gamma}^{\prime}=\lambda\left(\chi_{\gamma}(p)\right)$, and comparing unramified characters, we get $a_{p}^{\gamma}=\chi_{\gamma}(p) a_{p}$. Using the fact that $\chi_{\gamma}^{2}=\epsilon^{\gamma-1}$, we have $\chi_{\gamma}^{2}(p)=\epsilon^{\prime}(p)^{\gamma-1}$. Thus $\left(\mu^{f}\right)^{\gamma}=\mu^{f}$ and $\left(\nu^{f}\right)^{\gamma}=\nu^{f}$, since $\Gamma$ is abelian, so complex conjugation commutes with $\gamma$. Hence, $\gamma$ fixes $\mu^{f}+\nu^{f}$.

Now assume that $\chi_{\gamma}$ is ramified at $p$. Comparing ramified characters, we get, on $I_{p}$, that $\chi_{\gamma, p}=\epsilon_{p}^{\gamma}$ and $\epsilon_{p} \chi_{\gamma, p}=1$. Thus $\bar{\epsilon}_{p}=\epsilon_{p}^{\gamma}=\chi_{\gamma, p}$. Now, comparing unramified characters, we get $a_{p}^{\gamma}=\bar{a}_{p} \cdot \epsilon^{\prime}(p) \cdot \chi_{\gamma}^{\prime}(p)$. Again, since $\left(\chi_{\gamma}^{\prime}\right)^{2}=\left(\epsilon^{\prime}\right)^{\gamma-1}$, we deduce that

$$
\frac{\left(a_{p}^{2}\right)^{\gamma}}{\epsilon^{\prime}(p)^{\gamma}}=\frac{\bar{a}_{p}^{2}}{\bar{\epsilon}^{\prime}(p)}
$$

In other words, $\mu^{\gamma}=\nu$, and hence $\left(\mu^{f}\right)^{\gamma}=\nu^{f}$, for all integers $f \geq 1$. Applying complex conjugation we see that similarly $\left(\nu^{f}\right)^{\gamma}=\mu^{f}$. Hence again $\gamma$ fixes $\mu^{f}+\nu^{f}$.

In both cases $\gamma \in \Gamma=\operatorname{Gal}(E / F)$ is arbitrary, so $\mu^{f}+\nu^{f}$ must belong to $F$, for all integers $f \geq 1$. 
For later use we state the following generalization of Lemma 16 which can be proved in a similar manner, or directly by noting that $\alpha^{2} \equiv \epsilon \bmod F^{*}$.

Lemma 17: Let Frob $_{v}$ be an arithmetic Frobenius at $v$, and let $\zeta=\epsilon_{p}\left(\right.$ Frob $\left._{v}\right)$. Then $\mu^{f_{v}} \cdot 1 / \zeta+\nu^{f_{v}} \cdot \zeta \in F$.

6.1. UNEQUAL SLOPE. In this section, we assume that

$$
v\left(\frac{a_{p}^{2}}{\epsilon^{\prime}(p)}+2 p^{(k-1)}+\frac{\bar{a}_{p}^{2}}{\bar{\epsilon}^{\prime}(p)}\right)<k-1 .
$$

Here $v$ is the valuation such that $v(p)=1$.

By an elementary calculation it can be shown that the above assumption is equivalent to the assertion that for every place $w$ of $E$ lying over $v$, we have $w\left(a_{p}\right) \neq w\left(\bar{a}_{p}\right)$. Let $O_{v}$ be the ring of integers of $F_{v}$. Let $P_{v}$ be the prime ideal of $O_{v}$ and let $\pi_{v}$ be a prime element of $O_{v}$. Let $U_{v}^{(n)}=1+P_{v}^{n}$, for $n \geq 1$.

Lemma 18: $\mu$ and $\nu$ belong to $F_{v}$.

Proof. By Lemma 16, $\mu+\nu$ belongs to $F$. Consider the quantity

$$
\frac{(\mu-\nu)^{2}}{(\mu+\nu)^{2}}=1-4 \frac{\mu \cdot \nu}{(\mu+\nu)^{2}} \text {. }
$$

Now $\mu \nu=p^{2(k-1)}$. Since the slopes of $\mu$ and $\nu$ are not the same, the expression on the right-hand side belongs to $U_{v}^{(1)}=1+\pi_{v} O_{v}$, for $p$ odd, and it belongs to $U_{v}^{\left(3 e_{v}\right)}=1+\pi_{v}^{3 e_{v}} O_{v}$, for $p=2$. It therefore has a square root in $U_{v}^{(1)}=1+\pi_{v} O_{v}$, in both cases. Hence, $\frac{\mu-\nu}{\mu+\nu}$ belongs to $F_{v}$. Since we have already proved that $\mu+\nu$ belongs to $F$, we see $\mu-\nu$ belongs to $F_{v}$. Hence, individually, both $\mu$ and $\nu$ belong to $F_{v}$.

6.1.1. The case of odd primes. We now assume that $p$ is an odd prime. We say that $\epsilon_{p}$ is tame if the order of $\epsilon_{p}$ divides $p-1$.

Lemma 19: If $\epsilon_{p}$ is tame, then for any arithmetic Frobenius Frob $v$ at $v$,

$$
\frac{\left(a_{p}^{f_{v}}+\epsilon_{p}\left(\operatorname{Frob}_{v}\right)\left(\bar{a}_{p} \epsilon^{\prime}(p)\right)^{f_{v}}\right)^{2}}{a_{p}^{2 f_{v}}+\left(\bar{a}_{p} \epsilon^{\prime}(p)\right)^{2 f_{v}}} \in F_{v}^{* 2}
$$

is a square. 
Proof. We may rewrite this expression as

$$
\frac{\mu^{f_{v}}+\epsilon_{p}^{2}\left(\operatorname{Frob}_{v}\right) \nu^{f_{v}}}{\mu^{f_{v}}+\nu^{f_{v}}} \cdot\left(1+2 \epsilon_{p}\left(\operatorname{Frob}_{v}\right) \cdot \frac{p^{(k-1) f_{v}}}{\mu^{f_{v}}+\epsilon_{p}^{2}\left(\operatorname{Frob}_{v}\right) \nu^{f_{v}}}\right),
$$

where $\mu$ and $\nu$ are as above. By the previous lemma, $\mu$ and $\nu$ belong to $F_{v}$. Since $\epsilon_{p}$ is tame, the image of $\epsilon_{p}$ belongs to $\mathbb{Q}_{p}$, and hence to $F_{v}$. Thus all terms in the display above are in $F_{v}$. Now, since $p$ is odd, and the slopes are unequal, the second term (in parentheses) is in $U_{v}^{(1)}$, hence a square. If $w\left(a_{p}\right)>w\left(\bar{a}_{p}\right)$, the first term is of the form $\epsilon_{p}^{2}\left(\operatorname{Frob}_{v}\right)$ times an element of $U_{v}^{(1)}$, and if $w\left(a_{p}\right)<w\left(\bar{a}_{p}\right)$, then the first term is in $U_{v}^{(1)}$, so in both cases, the first term is also a square.

Lemma 20: If $\epsilon_{p}$ is tame and Frob $_{v}$ is an arithmetic Frobenius at $v$, then

$$
\alpha^{2}\left(\operatorname{Frob}_{v}\right) \equiv a_{p}^{2 f_{v}}+\left(\bar{a}_{p} \epsilon^{\prime}(p)\right)^{2 f_{v}} \bmod F_{v}^{* 2} .
$$

Proof. If the trace of $\rho_{f}(g)$ is non-zero, for $g \in G_{\mathbb{Q}}$, then (cf. part (4) of Proposition 8)

$$
\alpha^{2}(g) \equiv\left(\operatorname{trace} \rho_{f}(g)\right)^{2} \bmod F^{* 2} .
$$

Since $w\left(a_{p}\right) \neq w\left(\bar{a}_{p}\right)$, the trace of $\rho_{f}\left(\right.$ Frob $\left._{v}\right)$ is non-zero. Using Langlands' theorem to compute the trace, we obtain

$$
\alpha^{2}\left(\operatorname{Frob}_{v}\right) \equiv\left(a_{p}^{f_{v}}+\epsilon_{p}\left(\operatorname{Frob}_{v}\right)\left(\bar{a}_{p} \epsilon^{\prime}(p)\right)^{f_{v}}\right)^{2} \bmod F^{* 2} .
$$

The lemma now follows from the previous lemma.

Lemma 21: If $\epsilon_{p}$ is tame, then $\alpha(i)$ belongs to $F_{v}^{*}$, for $i \in I_{v}$.

Proof. If $i \in I_{v}$, and $\sigma_{v}$ is an arithmetic Frobenius at $v$, then $\sigma_{v}^{\prime}=\sigma_{v} i$ is also an arithmetic Frobenius at $v$. By the lemma above, $\alpha\left(\sigma_{v}\right) \equiv \pm \alpha\left(\sigma_{v}^{\prime}\right) \bmod F_{v}^{*}$. Since

$$
c_{\alpha}(\sigma, i)=\frac{\alpha\left(\sigma_{v}\right) \alpha(i)}{\alpha\left(\sigma_{v}^{\prime}\right)} \in F^{*},
$$

we see that $\alpha(i)$ belongs to $F_{v}^{*}$.

Theorem 22: Let $p$ be an odd prime such that $p \mid N$ and $N_{p}=C_{p}$. Let $v$ be a place of $F$ lying above $p$. Let $w$ be an extension of $v$ to a place of $E$. If $w\left(a_{p}\right) \neq w\left(\bar{a}_{p}\right)$, then $X_{v}$ is a matrix algebra if and only if

$$
m_{v}=\left[F_{v}: \mathbb{Q}_{p}\right] \cdot v\left(\mu+2 p^{k-1}+\nu\right) \in \mathbb{Z}
$$

is even, where $v$ is normalized so that $v(p)=1$. 
Proof. Let $L$ be the extension of $F_{v}$ cut out by the wild part of $\epsilon_{p}$. So $\epsilon_{p}$, thought of as a character of $G_{L}$, is tame. Note that $L / F_{v}$ is a totally ramified extension of odd (p-power) degree. By Lemma 21, $\tilde{\alpha}: G_{L} \rightarrow \bar{F}_{v}^{*} / F_{v}^{*}$ is an unramified character. On $G_{L}$, we have $\alpha^{2} \equiv \epsilon^{\prime} \bmod F_{v}^{*}$, since this is true with $\epsilon^{\prime}$ replaced with $\epsilon$, and on $G_{L}$ we have $\epsilon^{\prime} \equiv \epsilon \bmod F_{v}^{*}$, since $\epsilon_{p}\left(G_{L}\right) \subset \mathbb{Q}_{p}^{*} \subset F_{v}^{*}$, since $\left.\epsilon_{p}\right|_{G_{L}}$ is tame. We calculate $\operatorname{inv}_{L}\left(\operatorname{res}_{F_{v} / L} c_{\alpha}\right)$ using Lemma 9 applied to $K=\left.\alpha\right|_{G_{L}}$ and $t=\left.\epsilon^{\prime}\right|_{G_{L}}$. Let $u$ be the prime of $L$ lying over $v$ and let Frob $u$ be an arithmetic Frobenius at $u$. We obtain

$$
\operatorname{inv}_{L}\left(\operatorname{res}_{F_{v} / L} c_{\alpha}\right)=\frac{1}{2} \cdot u\left(\frac{\alpha^{2}\left(\operatorname{Frob}_{u}\right)}{\epsilon^{\prime}\left(\operatorname{Frob}_{u}\right)}\right) \bmod \mathbb{Z} \in{ }_{2} \operatorname{Br}(L) .
$$

Since $f_{v}$ is also the residue degree of $u \mid p$, by Lemma 20 we obtain

$$
\alpha^{2}\left(\operatorname{Frob}_{u}\right) \equiv a_{p}^{2 f_{v}}+\left(\bar{a}_{p} \epsilon^{\prime}(p)\right)^{2 f_{v}} \bmod F_{v}^{* 2} .
$$

Hence

$$
\frac{\alpha^{2}}{\epsilon^{\prime}}\left(\operatorname{Frob}_{u}\right) \equiv \mu^{f_{v}}+\nu^{f_{v}} \bmod F_{v}^{* 2} .
$$

Now $\left[L: F_{v}\right] \cdot \operatorname{inv}_{v}\left(c_{\alpha}\right)=\operatorname{inv}_{L}\left(\operatorname{res}_{F_{v} / L} c_{\alpha}\right)$, and for $x \in F_{v}, u(x)=\left[L: F_{v}\right] \cdot v(x)$, where both $u$ and $v$ are the surjective valuations onto $\mathbb{Z}$. But $\left[L: F_{v}\right]$ is a power of $p$, so is odd, and so in both cases can be ignored. We obtain $\operatorname{inv}_{v}\left(c_{\alpha}\right)=\frac{1}{2} \cdot v\left(\frac{\alpha^{2}}{\epsilon^{\prime}}\left(\operatorname{Frob}_{u}\right)\right)=\frac{1}{2} \cdot v\left(\mu^{f_{v}}+\nu^{f_{v}}\right)=\frac{1}{2} \cdot v\left(\mu^{f_{v}}+\nu^{f_{v}}+2 p^{(k-1) f_{v}}\right) \bmod \mathbb{Z}$. Since the last three terms lie in $F$ and have distinct valuations, replacing $v$ with the valuation $v$ satisfying $v(p)=1$, we obtain the theorem.

6.1.2. The case of $p=2$. We now assume that $p=2$, so that $N_{2}=C_{2} \geq 2$. We continue to assume that $w\left(a_{2}\right) \neq w\left(\bar{a}_{2}\right)$.

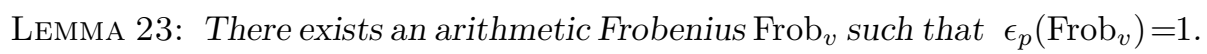

Proof. Let $\sigma_{v}$ be an arithmetic Frobenius at $v$. Then $\epsilon_{p}\left(\sigma_{v}\right)=\zeta_{2^{n}}$, a $2^{n}$-th root of unity, for $n \geq 0$. If $n=0$, we are done. Otherwise, since $\epsilon_{p}\left(G_{v}\right)=\epsilon_{p}\left(I_{v}\right)$, there exists $i \in I_{v}$ such that $\epsilon_{p}\left(\sigma_{v}^{2^{n}-1}\right)=\epsilon_{p}(i)$. Hence $\epsilon_{p}^{2 n}\left(\sigma_{v}\right)=1=\epsilon_{p}\left(\sigma_{v}\right)$. $\epsilon_{p}(i)=\epsilon_{p}\left(\tilde{\sigma}_{v}\right)$, where $\tilde{\sigma}_{v}=\sigma_{v} i$ is another arithmetic Frobenius at $v$.

Lemma 24: If $\operatorname{Frob}_{v}$ is an arithmetic Frobenius at $v$, then $\epsilon_{p}\left(\right.$ Frob $\left._{v}\right)$ belongs to $F_{v}^{*}$. 
Proof. Let $\sigma_{v}=\operatorname{Frob}_{v}$. Assume $\epsilon_{p}\left(\sigma_{v}\right)$ is a primitive $2^{m}$-th root of unity, for $m \geq 0$. Let $r \geq 1$ be such that $F_{v}$ contains a primitive $2^{r}$-th root of unity, but not a $2^{r+1}$-th root of unity. It is enough to prove $m \leq r$.

Assume, towards a contradiction, that $m \geq r+1$. Then $\epsilon_{p}^{2^{m-r-1}}\left(\sigma_{v}\right)$ is a $2^{r+1}$-th root of unity. Using the fact that $\epsilon_{p}\left(G_{v}\right)=\epsilon_{p}\left(I_{v}\right)$, we can find $i \in I_{v}$ such that $\epsilon_{p}^{2^{m-r-1}}\left(\sigma_{v}\right)=\epsilon_{p}\left(\sigma_{v} \cdot i\right)$ (see proof of previous lemma). For example, if $m=r+1$, we can take $i=1$. Now $\sigma_{v}^{\prime}=\sigma_{v} i$ is another arithmetic Frobenius at $v$. Using Langlands' theorem to compute the (non-zero) trace of $\rho_{f}\left(\sigma_{v}^{\prime}\right)$, we obtain

$$
\alpha\left(\sigma_{v}^{\prime}\right) \equiv a_{p}^{f_{v}}+\epsilon_{p}\left(\sigma_{v}^{\prime}\right)\left(\bar{a}_{p} \epsilon^{\prime}(p)\right)^{f_{v}} \bmod F^{*} .
$$

Since $\alpha^{2} \equiv \epsilon \bmod F^{*}$, we deduce that

$$
\frac{\mu^{f_{v}}+\epsilon_{p}^{2}\left(\sigma_{v}^{\prime}\right) \nu^{f_{v}}}{\epsilon_{p}\left(\sigma_{v}^{\prime}\right)} \in F^{*} .
$$

By Lemma $18, \mu^{f_{v}}$ and $\nu^{f_{v}}$ belong to $F_{v}$. Also, $\epsilon_{p}^{2}\left(\sigma_{v}^{\prime}\right)$ is a primitive $2^{r}$-th root of unity, so belongs to $F_{v}$. We conclude that the primitive $2^{r+1}$-th root of unity $\epsilon_{p}\left(\sigma_{v}^{\prime}\right)=\epsilon_{p}^{2^{m-r-1}}\left(\sigma_{v}\right)$ belongs to $F_{v}$, a contradiction.

Lemma 25: If $i \in I_{v}$, then $\alpha(i)$ belongs to $F_{v}^{*}$.

Proof. If $\epsilon_{p}\left(G_{v}\right)= \pm 1$, then by Langlands' theorem

$$
\alpha\left(\operatorname{Frob}_{v}\right) \equiv a_{p}^{f_{v}} \pm\left(\bar{a}_{p} \epsilon^{\prime}(p)\right)^{f_{v}} \bmod F^{*} .
$$

Let $i$ be an arbitrary element of $I_{v}$ and let $\sigma_{v}$ and $\sigma_{v}^{\prime}=\sigma_{v} i$ be two arithmetic Frobenii at $v$. The above congruence for $\alpha$ (and a calculation similar to that in Lemma 18 and Lemma 20 in the case of unequal sign) guarantees that $\alpha\left(\sigma_{v}\right) \equiv$ $\alpha\left(\sigma_{v}^{\prime}\right) \bmod F_{v}^{*}$. Since $\alpha\left(\sigma_{v}\right) \alpha(i) / \alpha\left(\sigma_{v}^{\prime}\right) \in F^{*}$, so $\alpha(i) \in F_{v}$.

Let us assume now that $\epsilon_{p}\left(G_{v}\right) \neq \pm 1$. We first show that if $\epsilon_{p}(i) \neq-1$, then $\alpha(i) \in F_{v}$. We first choose an arithmetic Frobenius $\sigma_{v}$ such that $\epsilon_{p}\left(\sigma_{v}\right)=1$, by Lemma 23. Then $\epsilon_{p}(i)=\epsilon_{p}\left(\sigma_{v}\right) \cdot \epsilon_{p}(i)=\epsilon_{p}\left(\sigma_{v}^{\prime}\right)$, for $\sigma_{v}^{\prime}=\sigma_{v} i$. Hence $\epsilon_{p}(i) \in F_{v}$, by Lemma 24. By Langlands' theorem, we know $\alpha(i) \equiv 1+\epsilon_{p}(i) \bmod F^{*}$. Hence, $\alpha(i)$ belongs to $F_{v}^{*}$. If $\epsilon_{p}(i)=-1$, we choose $j \in I_{v}$ such that $\epsilon_{p}(j) \neq \pm 1$, using the fact that $\epsilon_{p}\left(G_{v}\right)=\epsilon_{p}\left(I_{v}\right)$. Since $\epsilon_{p}(j)$ and $\epsilon_{p}(i j) \neq-1$, the previous argument shows that $\alpha(j)$ and $\alpha(i j)$ belongs to $F_{v}$. Since $\alpha(i) \alpha(j) / \alpha(i j) \in F^{*}$, we see that $\alpha(i) \in F_{v}^{*}$. 
Lemma 26: Let Frob $v$ be an arithmetic Frobenius at $v$. Then

$$
\alpha^{2}\left(\operatorname{Frob}_{v}\right) \equiv a_{p}^{2 f_{v}}+\left(\bar{a}_{p} \epsilon^{\prime}(p)\right)^{2 f_{v}}+2 p^{(k-1) f_{v}} \epsilon^{\prime}(p)^{f_{v}} \bmod F_{v}^{* 2} .
$$

Proof. Let $\sigma_{v}$ be a Frobenius as in Lemma 23, and let $\tilde{\sigma}_{v}$ be any arithmetic Frobenius at $v$. Then $\sigma_{v}$ and $\tilde{\sigma}_{v}$ will differ by an element of $I_{v}$. By Lemma 25,

$$
\alpha\left(\tilde{\sigma}_{v}\right) \equiv \alpha\left(\sigma_{v}\right) \bmod F_{v}^{*} .
$$

Since $\epsilon_{p}\left(\sigma_{v}\right)=1$, we get by Langlands' theorem

$$
\alpha^{2}\left(\sigma_{v}\right) \equiv a_{p}^{2 f_{v}}+\left(\bar{a}_{p} \epsilon^{\prime}(p)\right)^{2 f_{v}}+2 p^{(k-1) f_{v}} \epsilon^{\prime}(p)^{f_{v}} \bmod F^{* 2} .
$$

Hence,

$$
\alpha^{2}\left(\tilde{\sigma}_{v}\right) \equiv a_{p}^{2 f_{v}}+\left(\bar{a}_{p} \epsilon^{\prime}(p)\right)^{2 f_{v}}+2 p^{(k-1) f_{v}} \epsilon^{\prime}(p)^{f_{v}} \bmod F_{v}^{* 2} .
$$

Theorem 27: Let $p=2$ and assume $N_{2}=C_{2} \geq 1$. Let $v \mid 2$ be a place of $F$. Assume that $w\left(a_{2}\right) \neq w\left(\bar{a}_{2}\right)$. Then $X_{v}$ is a matrix algebra over $F_{v}$ if and only if

$$
m_{v}=\left[F_{v}: \mathbb{Q}_{2}\right] \cdot v\left(\mu+2 p^{k-1}+\nu\right) \in \mathbb{Z}
$$

is even, where $v$ is normalized such that $v(p)=1$.

Proof. By Lemma 25, the map $\alpha: G_{v} \longrightarrow \bar{F}_{v}^{*} / F_{v}^{*}$ is unramified. Applying Lemma 9 with $K=\left.\alpha\right|_{G_{v}}$ and $t=\left.\epsilon^{\prime}\right|_{G_{v}}$, we have

$$
\operatorname{inv}_{v}\left(c_{\alpha}\right)=\frac{1}{2} \cdot v\left(\frac{\alpha^{2}}{\epsilon^{\prime}}\left(\operatorname{Frob}_{v}\right)\right)=\frac{1}{2} \cdot v\left(\mu^{f_{v}}+2 p^{(k-1) f_{v}}+\nu^{f_{v}}\right) \bmod \mathbb{Z},
$$

where the last equality follows from Lemma 26. The theorem now follows replacing $v$ by the valuation $v$ normalized such that $v(p)=1$.

6.2. EQUAL SLOPE. In this section, we assume that

$$
v\left(\frac{a_{p}^{2}}{\epsilon^{\prime}(p)}+2 p^{(k-1)}+\frac{\bar{a}_{p}^{2}}{\bar{\epsilon}^{\prime}(p)}\right) \geq k-1,
$$

where $v(p)=1$. So $w\left(a_{p}\right)=w\left(\bar{a}_{p}\right)$, for every place $w$ of $E$ lying above $v$. In this case it is possible for $m_{v}=\infty$. To avoid this we introduce a new quantity $m_{v}^{\zeta}$, for any root of unity $\zeta$ in the image of $\epsilon_{p}$, defined by

$$
m_{v}^{\zeta}:=e_{v} \cdot v\left(\mu^{f_{v}} \cdot 1 / \zeta+2 p^{(k-1) f_{v}}+\nu^{f_{v}} \cdot \zeta\right) \in \mathbb{Z} \cup\{\infty\},
$$

where $v$ is normalized such that $v(p)=1$. By Lemma 17, the three-term expression above is in $F$, so the above expression is well-defined. Moreover, for 
some $\zeta$, the three-term expression above is non-zero and $m_{v}^{\zeta} \in \mathbb{Z}$ is finite. When $\zeta \in F_{v}^{*}$, e.g., if $\zeta$ is the value of the tame part of $\epsilon_{p}$, then we may rewrite

$$
m_{v}^{\zeta}=e_{v} \cdot v\left(\mu^{f_{v}}+2 \zeta p^{(k-1) f_{v}}+\zeta^{2} \nu^{f_{v}}\right) \in \mathbb{Z} \cup\{\infty\} .
$$

Note that in the unequal slope case $m_{v}^{\zeta}=m_{v}$, if $\zeta \in F_{v}^{*}$, so the quantities $m_{v}^{\zeta}$ may be considered as generalizations of $m_{v}$ in the equal slope case. In particular, taking $\zeta= \pm 1$ we have

$$
m_{v}^{ \pm}=e_{v} \cdot v\left(\mu^{f_{v}} \pm 2 p^{(k-1) f_{v}}+\nu^{f_{v}}\right) \in \mathbb{Z} \cup\{\infty\} .
$$

We remark that $m_{v}^{+}$is finite if and only if $a_{p}^{f_{v}}+\left(\bar{a}_{p} \epsilon^{\prime}(p)\right)^{f_{v}} \neq 0$, and $m_{v}^{-}$is finite if and only if $a_{p}^{f_{v}}-\left(\bar{a}_{p} \epsilon^{\prime}(p)\right)^{f_{v}} \neq 0$, so that one of the two quantities $m_{v}^{ \pm}$ is always finite.

6.2.1. The case of odd primes. We now assume that $p$ is odd and work under a condition on the tame part of $\epsilon_{p}$.

Theorem 28: Let $p$ be an odd prime with $N_{p}=C_{p} \geq 1$ and $v \mid p$ be a place of $F$. Assume that the tame part of $\epsilon_{p}$ on $G_{v}$ is not quadratic. Let $\zeta$ be in the image of the tame part of $\epsilon_{p}$ on $G_{v}$. Then the parity of

$$
m_{v}^{\zeta}=e_{v} \cdot v\left(\mu^{f_{v}}+2 \zeta p^{(k-1) f_{v}}+\zeta^{2} \nu^{f_{v}}\right) \in \mathbb{Z} \cup\{\infty\}
$$

is independent of $\zeta$ when it is finite, and then $X_{v}$ is a matrix algebra over $F_{v}$ if and only if $m_{v}^{\zeta} \in \mathbb{Z}$ is even.

Thus, if -1 lies in the image of the tame part of $\epsilon_{p}$ on $G_{v}$, and

- if $a_{p}^{f_{v}}+\left(\bar{a}_{p} \epsilon^{\prime}(p)\right)^{f_{v}} \neq 0$, then $X_{v}$ is a matrix algebra over $F_{v}$ if and only if $m_{v}^{+} \in \mathbb{Z}$ is even,

- if $a_{p}^{f_{v}}-\left(\bar{a}_{p} \epsilon^{\prime}(p)\right)^{f_{v}} \neq 0$, then $X_{v}$ is a matrix algebra over $F_{v}$ if and only $m_{v}^{-} \in \mathbb{Z}$ is even,

noting that one of $m_{v}^{ \pm}$is always finite, and both have the same parity if both are finite. If -1 does not lie in the image of the tame part of $\epsilon_{p}$ on $G_{v}$ (e.g., if the tame part of $\epsilon_{p}$ is trivial on $G_{v}$ ) and if $m_{v}^{+}<\infty$, then $X_{v}$ is a matrix algebra over $F_{v}$ if and only if $m_{v}^{+} \in \mathbb{Z}$ is even.

Proof. The proof goes along the lines of the proof of Theorem 22, with a few modifications. We base change to $L$ so that $\left.\epsilon_{p}\right|_{G_{L}}$ is tame, compute the invariant there, and then descend back to $F_{v}$. 
We first show that $\tilde{\alpha}: G_{L} \rightarrow \bar{F}_{v}^{*} / F_{v}^{*}$ is unramified. If the trace of $\rho_{f}(g)$ is non-zero, then $\alpha(g) \equiv \operatorname{trace} \rho_{f}(g) \bmod F^{*}$, for $g \in G_{L}$ (cf. part (4) of Proposition 8). If the tame part of $\epsilon_{p}$ is trivial on $G_{v}$, then by Langlands' theorem $\alpha(i) \equiv 1+\epsilon_{p}(i)=2 \bmod F^{*}$, for all $i \in I_{L}$. So we may assume that the tame part of $\epsilon_{p}$ is non-trivial on $G_{v}$. If $\epsilon_{p}(i) \neq-1$, for $i \in I_{L}$, then $\alpha(i)$ belongs to $F_{v}^{*}$. Indeed by Langlands' theorem again, $\alpha(i) \equiv 1+\epsilon_{p}(i) \bmod$ $F^{*}$, and since $\epsilon_{p}$ is tame on $G_{L}, \epsilon_{p}(i) \in \mathbb{Q}_{p}^{*} \subset F_{v}^{*}$. If $\epsilon_{p}(i)=-1$, for $i \in I_{L}$, we choose $j \in I_{L}$ such that $\epsilon_{p}(j) \neq \pm 1$. Such a choice is possible since by assumption the tame part of $\epsilon_{p}$ is not quadratic. The above argument shows that $\alpha(j)$ and $\alpha(i j)$ belong to $F_{v}^{*}$, and since $\alpha(i) \alpha(j) / \alpha(i j) \in F^{*}, \alpha(i) \in F_{v}^{*}$ as well.

Write $u$ for the prime of $L$ lying over $v$ and let $\operatorname{Frob}_{u}$ be an arithmetic Frobenius at $u$. We calculate $\operatorname{inv}_{L}\left(\operatorname{res}_{F_{v} / L} c_{\alpha}\right)$ using Lemma 9 applied to $K=$ $\left.\alpha\right|_{G_{L}}$ and $t=\left.\epsilon^{\prime}\right|_{G_{L}}$, and get

$$
\operatorname{inv}_{L}\left(\operatorname{res}_{F_{v} / L} c_{\alpha}\right)=\frac{1}{2} \cdot u\left(\frac{\alpha^{2}}{\epsilon^{\prime}}\left(\operatorname{Frob}_{u}\right)\right) \bmod \mathbb{Z}
$$

Since $\left[L: F_{v}\right]$ is odd (a power of $p$ ), we may descend to $F_{v}$ as before to get

$$
\operatorname{inv}_{v}\left(c_{\alpha}\right)=\frac{1}{2} \cdot v\left(\frac{\alpha^{2}}{\epsilon^{\prime}}\left(\operatorname{Frob}_{u}\right)\right) \bmod \mathbb{Z} .
$$

Let $\zeta=\epsilon_{p}\left(\operatorname{Frob}_{u}\right) \in \mathbb{Q}_{p}^{*} \subset F_{v}^{*}$. Then the usual argument using Langlands' theorem shows that

$$
\frac{\alpha^{2}}{\epsilon^{\prime}}\left(\operatorname{Frob}_{u}\right) \equiv \mu^{f_{v}}+2 \zeta p^{(k-1) f_{v}}+\zeta^{2} \nu^{f_{v}} \bmod F_{v}^{* 2}
$$

and replacing $v$ with the valuation $v$ such that $v(p)=1$ we obtain the theorem. We note that the parity of $m_{v}^{\zeta}$ is independent of $\zeta$ since $\tilde{\alpha}$ is unramified on $G_{L}$.

6.2.2. The case of $p=2$. We now show that if $p=2$ and $\epsilon_{2}$ is not quadratic on $G_{v}$, then the ramification of $X_{v}$ is also determined by $m_{v}^{ \pm}$, up to an error term $n_{v}$ which depends purely on the nebentypus $\epsilon_{2}$, which we define now.

If $\epsilon_{2}$ is trivial on $G_{v}$, set $n_{v}=0$. If $\epsilon_{2}$ has order $2^{r}$ on $G_{v}$, for $r>1$, let $F_{v}(\sqrt{t}) / F_{v}$, for $t \in F_{v}^{*}$, be the quadratic extension of $F_{v}$ cut out by the quadratic character $\epsilon_{2}^{r-1}$ on $G_{v}$. Let $\zeta_{2^{r}}$ be a primitive $2^{r}$-th root of unity and define

$$
z=\frac{\left(1+\zeta_{2^{r}}\right)^{2}}{\zeta_{2^{r}}} \in F^{*}
$$


noting that $z \in F^{*}$ by Langlands' theorem. Define $n_{v} \bmod 2$ by

$$
(-1)^{n_{v}}=\epsilon_{v}(-1) \cdot(t, z)_{v},
$$

where $\epsilon_{v}$ is the restriction of $\epsilon_{2}$ to $G_{v}$ and $(t, z)_{v}$ is the Hilbert symbol of $t$ and $z$ at $v$.

Lemma 29: Assume $\epsilon_{2}$ has order $2^{r}$ on $G_{v}$, for $r>1$. Let $h$ be the function on $G_{v}$ defined by

$$
h(g)= \begin{cases}\frac{1+\epsilon_{2}(g)}{\sqrt{\epsilon_{2}(g)}} & \text { if } \epsilon_{2}(g) \neq-1, \\ 1 & \text { if } \epsilon_{2}(g)=-1\end{cases}
$$

and let $c_{h}$ be the corresponding $F$-valued 2-cocycle on $G_{v}$. Then the class of $c_{h}$ in ${ }_{2} \operatorname{Br}\left(F_{v}\right)$ is given by the symbol $(t, z)_{v}$.

Proof. We first claim that if $-1 \neq \zeta=\epsilon_{2}(g)$ is not a primitive $2^{r}$-th root of unity, then $\frac{1+\zeta}{\sqrt{\zeta}} \in F^{*}$. Indeed, choose $g \in G_{v}$ such that $\epsilon_{2}(g)=\zeta_{2^{r}}$, where $\zeta_{2^{r}}$ is a primitive $2^{r}$-th root of unity. We may assume $g \in I_{v}$, and applying Langlands' theorem we obtain that $\frac{\left(1+\epsilon_{2}(g)\right)^{2}}{\epsilon_{2}(g)} \in F^{*}$, and hence that $\frac{1+\zeta_{2^{r-1}}}{\sqrt{\zeta_{2^{r-1}}}} \in F^{*}$, where $\zeta_{2^{r-1}}=\epsilon_{2}\left(g^{2}\right)$ is a primitive $2^{r-1}$-th root of unity. Now set $h=g^{2} \in I_{v}$. Set $d=\frac{\alpha^{2}}{\epsilon_{2}}$ on $I_{v}$. Then by Langlands' theorem $d(h) \in F^{* 2}$. Since $d: I_{v} \rightarrow F^{*} / F^{* 2}$ is a homomorphism we see that $d\left(h^{a}\right) \in F^{* 2}$, for all integers $a$. Hence by Langlands' theorem again we deduce that $\frac{\left(1+\zeta_{2 r-1}^{a}\right)^{2}}{\zeta_{2^{r-1}}^{a}} \in F^{* 2}$, if it is non-zero. Hence $\frac{1+\zeta_{2^{r-1}}^{a}}{\sqrt{\zeta_{2^{r-1}}^{a}}} \in F^{*}$, for all integers $a$, if it is non-zero, proving the claim. We now claim that if $\epsilon_{2}\left(g^{b}\right)$ with $b$ odd is any primitive $2^{r}$-th root of unity, then $h\left(g^{b}\right) \equiv h(g) \bmod F^{*}$. Indeed by the discussion above $h\left(g^{b-1}\right) \in F^{*}$ since $b-1$ is even.

The two claims above show that the 2-cocycle $c_{h}$ is cohomologous to the 2-cocycle $c_{l}$ where

$$
l(g)= \begin{cases}1 & \text { if } \epsilon_{2}^{2^{r-1}}(g)=1 \\ \frac{1+\zeta_{2^{r}}}{\sqrt{\zeta_{2}}} & \text { if } \epsilon_{2}^{2^{r-1}}(g)=-1\end{cases}
$$


Let $\sigma$ be the non-trivial element of the Galois group $\operatorname{Gal}\left(F_{v}(\sqrt{t}) / F_{v}\right)$. Let $z=\left(\frac{1+\zeta_{2} r}{\sqrt{\zeta_{2} r}}\right)^{2} \in F^{*}$. Then the class of $c_{l}$ is completely determined by the table

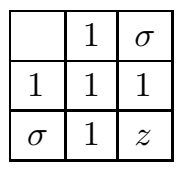

which is precisely the symbol $(t, z)_{v}$.

Theorem 30: Let $p=2$ and assume that $\epsilon_{2}$ is not quadratic on $G_{v}$. If $\epsilon_{2}$ is trivial on $G_{v}$ and $m_{v}^{+}<\infty$, then $X_{v}$ is a matrix algebra if and only if $m_{v}^{+} \in \mathbb{Z}$ is even. If $\epsilon_{2}$ on $G_{v}$ has order 4 or more and if

- $a_{p}^{f_{v}}+\left(\bar{a}_{p} \epsilon^{\prime}(p)\right)^{f_{v}} \neq 0$, then $X_{v}$ is a matrix algebra if and only if $m_{v}^{+}+n_{v} \in$ $\mathbb{Z}$ is even,

- $a_{p}^{f_{v}}-\left(\bar{a}_{p} \epsilon^{\prime}(p)\right)^{f_{v}} \neq 0$, then $X_{v}$ is a matrix algebra if and only if $m_{v}^{-}+n_{v} \in$ $\mathbb{Z}$ is even,

noting that if both $a_{p}^{f_{v}} \pm\left(\bar{a}_{p} \epsilon^{\prime}(p)\right)^{f_{v}} \neq 0$, then $m_{v}^{ \pm}$have the same parity.

Proof. If $\epsilon_{2}$ is trivial on $G_{v}$, then by Langlands' theorem, $\alpha(i) \equiv 2 \bmod F^{*}$, for all $i \in I_{v}$. Lemma 9 applies directly to prove the first statement. So we may assume that $\epsilon_{2}$ is not of order 1 or order 2 on $G_{v}$. Hence there exists $i \in I_{v}$, such that $\epsilon_{2}(i)=\sqrt{-1}$. If $\epsilon_{2}(j)=-1$, for $j \in I_{v}$, then a short computation using the fact that $c_{\alpha}(i, j) \in F^{*}$ shows that $\alpha(j) \equiv \sqrt{-1} \bmod F^{*}$.

We define a function $f: G_{v} \rightarrow E^{*}$ by

$$
f(g)= \begin{cases}1+\epsilon_{2}(g) & \text { if } \epsilon_{2}(g) \neq-1 \\ \sqrt{-1} & \text { if } \epsilon_{2}(g)=-1\end{cases}
$$

Now define $K: G_{v} \rightarrow E^{*}$ by $K(g)=\frac{\alpha(g)}{f(g)}$, for $g \in G_{v}$. Then the cocycle $c_{\alpha}$ can be decomposed as $c_{\alpha}=c_{K} c_{f}$, where $c_{K}$ and $c_{f}$ are the cocycles corresponding to $K$ and $f$ respectively. That these are indeed cocycles follows from the fact that they are $F$-valued, which can be proved using $\epsilon_{2}\left(G_{v}\right)=\epsilon_{2}\left(I_{v}\right)$ and Langlands' theorem.

We first calculate $\operatorname{inv}_{v}\left(c_{K}\right)$. By choice of $f, K(i)$ belongs to $F^{*}$, for all $i \in I_{v}$. Since $\epsilon_{2}\left(G_{v}\right)=\epsilon_{2}\left(I_{v}\right)$, a computation using Langlands' theorem shows that $\frac{K^{2}(g)}{\epsilon^{\prime}(g)} \in F^{*}$, for all $g \in G_{v}$. Let $\sigma_{v}$ be the Frobenius at the prime $v$. By 
Lemma 9 applied to $K$ as above and $t=\epsilon^{\prime}$ we have

$$
\operatorname{inv}_{v}\left(c_{K}\right)=\frac{1}{2} \cdot v\left(\frac{K^{2}}{\epsilon^{\prime}}\left(\sigma_{v}\right)\right) \bmod \mathbb{Z}
$$

Assume $a_{2}^{f_{v}} \neq-\left(\bar{a}_{2} \epsilon^{\prime}(2)\right)^{f_{v}}$; then we choose $\sigma_{v}$ in such a way that $\epsilon_{2}\left(\sigma_{v}\right)=1$. Then $\alpha\left(\sigma_{v}\right) \equiv\left(a_{2}^{f_{v}}+\left(\bar{a}_{2} \epsilon^{\prime}(2)\right)^{f_{v}}\right) \bmod F^{*}$, so that $\frac{K^{2}}{\epsilon^{\prime}}\left(\sigma_{v}\right) \equiv \mu^{f_{v}}+\nu^{f_{v}}+2 p^{(k-1) f_{v}}$ $\bmod F^{* 2}$. Finally, the valuation considered in the statement of the theorem is normalized so that $v(2)=1$, and differs from the valuation used in the proof by $e_{v}$. Noting $e_{v} f_{v}=\left[F_{v}: \mathbb{Q}_{2}\right]$, we obtain

$$
\operatorname{inv}_{v}\left(c_{K}\right)=\frac{1}{2} \cdot m_{v}^{+} \bmod \mathbb{Z}
$$

If $a_{2}^{f_{v}}=-\left(\bar{a}_{2} \epsilon^{\prime}(2)\right)^{f_{v}}$, then we choose $\sigma_{v}$ in such a way that $\epsilon_{2}\left(\sigma_{v}\right)=-1$. Then $\alpha\left(\sigma_{v}\right) \equiv\left(a_{2}^{f_{v}}-\left(\bar{a}_{2} \epsilon^{\prime}(2)\right)^{f_{v}}\right) \bmod F^{*}$, so that $\frac{K^{2}}{\epsilon^{\prime}}\left(\sigma_{v}\right) \equiv \mu^{f_{v}}+\nu^{f_{v}}-2 p^{(k-1) f_{v}}$ $\bmod F^{* 2}$. We obtain

$$
\operatorname{inv}_{v} c_{K}=\frac{1}{2} \cdot m_{v}^{-} \bmod \mathbb{Z}
$$

We also remark that since $K^{2} / \epsilon^{\prime}: G_{v} \rightarrow F^{*} / F^{* 2}$ is an unramified homomorphism, $\operatorname{inv}_{v}\left(c_{K}\right)$ does not depend on the choice of arithmetic Frobenius at $v$, and in particular $m_{v}^{ \pm}$have the same parity if both are simultaneously finite.

Now we calculate $\operatorname{inv}_{v}\left(c_{f}\right)$. Let $c_{\epsilon}$ be the cocycle

$$
c_{\epsilon}(g, h)=\frac{\sqrt{\epsilon(g)} \sqrt{\epsilon(h)}}{\sqrt{\epsilon(g h)}},
$$

for $g, h \in G_{\mathbb{Q}}$. We have $\operatorname{inv}_{v}\left(c_{f}\right)=\operatorname{inv}_{v}\left(c_{h}\right)+\operatorname{inv}_{v}\left(c_{\epsilon}\right)$, where $h$ is the function defined in the previous lemma. The theorem now follows from the previous lemma and the fact that $\operatorname{inv}_{v}\left(c_{\epsilon}\right)$ is trivial if and only if $\epsilon_{v}(-1)=1$ [Qu98].

Corollary 31: Assume that $p=2$ and $\epsilon_{2}$ is not quadratic on $G_{v}$. Assume also that $F=\mathbb{Q}$. Then:

(1) If $\epsilon_{2}(-1)=1$, then $X_{v}$ is a matrix algebra over $F_{v}$ if and only if one of $m_{v}^{ \pm} \in \mathbb{Z}$ is even.

(2) If $\epsilon_{2}(-1)=-1$, then $X_{v}$ is a matrix algebra over $F_{v}$ if and only if one of $m_{v}^{ \pm} \in \mathbb{Z}$ is odd.

Proof. We show that the symbol $(t, z)_{v}$ vanishes when $F=\mathbb{Q}$, so that $n_{v}=0$ $\bmod 2$ if and only if $\epsilon_{2}(-1)=1$. Note that $\mathbb{Q}$ has three quadratic extensions of absolute discriminant a power of 2 , namely $\mathbb{Q}(i), \mathbb{Q}(\sqrt{2})$ and $\mathbb{Q}(\sqrt{-2})$, but only 
the middle one is cut out by the quadratic character $\epsilon_{2}^{2^{r-1}}$, since it is an even character of level 8. It follows that $t=2 \in \mathbb{Q}_{2}$ and $(t, z)_{v}=\left(t, N_{F_{v} / \mathbb{Q}_{2}}(z)\right)_{2}$.

To compute the norm, we assume $F$ is general. Now $N_{\mathbb{Q}\left(\zeta_{2^{r}}\right) / \mathbb{Q}}\left(\zeta_{2 r}\right)=1$ and $N_{\mathbb{Q}\left(\zeta_{2^{r}}\right) / \mathbb{Q}}\left(1+\zeta_{2^{r}}\right)=2$ for all $r>1$, since the minimal polynomial of $1+\zeta_{2^{r}}$ is $(x-1)^{2^{r-1}}+1=0$. Since the local norm is the same as the global norm, we have $N_{\mathbb{Q}_{2}\left(\zeta_{2^{r}}\right) / \mathbb{Q}_{2}}(z)=4$. We have $\mathbb{Q}_{2} \subset \mathbb{Q}(z)_{v} \subset \mathbb{Q}_{2}\left(\zeta_{2^{r}}\right)$. Noting that the second index is 2 , by the transitivity of the norm in towers we have $N_{\mathbb{Q}(z)_{v} / \mathbb{Q}_{2}}(z)^{2}=4$, and we obtain $N_{\mathbb{Q}(z)_{v} / \mathbb{Q}_{2}}(z)= \pm 2$. Since $z \in F$, by the transitivity of the norm again, we obtain $N_{F_{v} / \mathbb{Q}_{2}}(z)=( \pm 2)^{d}$ where $d=\left[F_{v}: \mathbb{Q}(z)_{v}\right]$.

Thus $(t, z)_{v}=\left(t, N_{F_{v} / \mathbb{Q}_{2}}(z)\right)_{2}=(2, \pm 2)_{2}^{d}=1$, since $(2, \pm 2)_{2}=1$, as one can check directly.

The corollary predicts that when $p=2$ and $F=\mathbb{Q}$, there is a switch in the parity of $m_{v}^{ \pm}$in determining the triviality of the class of $X_{v}$, when $\epsilon_{2}$ moves from even to odd characters. For a numerical example of this interesting phenomenon, see Example 5 at the end of the paper.

6.2.3. Remaining quadratic cases. If the tame part of $\epsilon_{p}$ is quadratic on $G_{v}$ for an odd prime $p$ or if $p=2$ and $\epsilon_{2}$ is quadratic on $G_{v}$, we again show that $X_{v}$ is determined completely by $m_{v}^{ \pm}$up to an extra Hilbert symbol. The following results are quite general and hold for the unequal slope case also. In the case of unequal slope the extra symbol is trivial.

We need some notation.

Assume that the quadratic extension cut out by the tame part of $\epsilon_{p}$ if $p$ is odd, or by $\epsilon_{p}$ if $p=2$, is $F_{v}(\sqrt{t})$, for some $t \in F_{v}^{*}$.

Define

$$
a=\frac{\mu^{f_{v}}+\nu^{f_{v}}+2 p^{(k-1) f_{v}}}{\mu^{f_{v}}+\nu^{f_{v}}-2 p^{(k-1) f_{v}}} \in F^{*} \cup\{0, \infty\} .
$$

Note $a \in F^{*}$ if and only if $a_{p}^{f_{v}} \neq \pm\left(\bar{a}_{p} \epsilon^{\prime}(p)\right)^{f_{v}}$. In this case define the integer $n_{v}$ $\bmod 2$ by $(-1)^{n_{v}}=(t, a)_{v}$. Let $p^{\dagger} \nmid N$ be an auxiliary prime such that $a_{p^{\dagger}} \neq 0$ and such that, for all $\gamma \in \Gamma$,

$$
\chi_{\gamma}\left(p^{\dagger}\right)= \begin{cases}-1 & \text { if } \chi_{\gamma} \text { is ramified at } p \\ 1 & \text { if } \chi_{\gamma} \text { is unramified at } p\end{cases}
$$


We can always choose $p^{\dagger}$ as above, since $f$ is a non-CM form. Since $\epsilon^{-1}$ is an extra twist, we have $\epsilon\left(p^{\dagger}\right)=-1$. Let

$$
b=a_{p^{\dagger}}^{2}=-\frac{a_{p^{\dagger}}^{2}}{\epsilon\left(p^{\dagger}\right)} \in F^{*} .
$$

If $a_{p}^{f_{v}}=\left(\bar{a}_{p} \epsilon^{\prime}(p)\right)^{f_{v}}$, define an integer $n_{v} \bmod 2$ by $(-1)^{n_{v}}=(t, b)_{v}$, and if $a_{p}^{f_{v}}=-\left(\bar{a}_{p} \epsilon^{\prime}(p)\right)^{f_{v}}$, define $n_{v}$ by $(-1)^{n_{v}}=(t, b)_{v} \cdot(-1)^{\left(e_{v} v(b)\right)}$.

Theorem 32: Assume that the tame part of $\epsilon_{p}$ is quadratic for an odd prime $p$, or $p=2$ and $\epsilon_{2}$ is quadratic.

(1) Assume that $a \in F^{*}$. Then, $X_{v}$ is a matrix algebra over $F_{v}$ if and only if

$$
m_{v}^{+}+n_{v}
$$

is even.

(2) If $a_{p}^{f_{v}}=\left(\bar{a}_{p} \epsilon^{\prime}(p)\right)^{f_{v}}$, then $X_{v}$ is a matrix algebra over $F_{v}$ if and only if

$$
m_{v}^{+}+n_{v}
$$

is even.

(3) If $a_{p}^{f_{v}}=-\left(\bar{a}_{p} \epsilon^{\prime}(p)\right)^{f_{v}}$, then $X_{v}$ is a matrix algebra over $F_{v}$ if and only if

$$
m_{v}^{-}+n_{v}
$$

is even.

Proof. If $\epsilon_{p}(i)=-1$, for $i \in I_{v}$, then $\alpha^{2}(i) \in F^{*}$. We claim that the image of $\alpha(i)^{2}$ in $F_{v}^{*} / F_{v}^{* 2}$ is constant, i.e., there exists $d \in F_{v}^{*}$ such that $\alpha^{2}(i) \equiv d$ $\bmod F_{v}^{* 2}$. Indeed, a priori $\alpha(i)=\sqrt{t(i)} d(i)$, for some $t(i), d(i) \in F_{v}^{*}$. If $j \in I_{v}$ with $\epsilon_{p}(j)=-1$, then by Langlands' theorem, since $\epsilon_{p}(i j)=1, \alpha(i j) \in F^{*}$. Since $c_{\alpha}(i, j) \in F^{*}$, we get $\sqrt{t(i)} \equiv \sqrt{t(j)} \bmod F_{v}^{*}$, as desired. Thus $\sqrt{t(i)} \equiv$ $\sqrt{d} \bmod F_{v}^{*}$ for all $i \in I_{v}$ such that $\epsilon_{p}(i)=-1$. We compute $d$ and show that the ramification of $X_{v}$ is controlled by $m_{v}^{ \pm}$, and an extra Hilbert symbol involving $d$. In case (1) we show we can take $d=a$, whereas in case (2) and (3) we show we can take $d=b$.

For $p$ odd, we do a base change as in Theorem 22 and assume without loss of generality that $\epsilon_{p}$ is tame (and quadratic).

Assume we are in case (1), so that $a \in F^{*}$. Let $\sigma_{v}$ be an arithmetic Frobenius at $v$, such that $\epsilon_{p}\left(\sigma_{v}\right)=1$. Let $i \in I_{v}$ be such that $\epsilon_{p}(i)=-1$. By Langlands' 
theorem,

$$
\frac{\alpha\left(\sigma_{v}\right)}{\alpha\left(\sigma_{v} i\right)} \equiv \sqrt{a} \bmod F^{*}
$$

Since $c_{\alpha}\left(\sigma_{v}, i\right) \in F^{*}$, and $a$ belongs to $F^{*}$, we have $\alpha(i) \equiv \sqrt{a} \bmod F^{*}$. We define a function $f$ on $G_{v}$ by

$$
f(g)= \begin{cases}1 & \text { if } \epsilon_{p}(g)=1 \\ \sqrt{a} & \text { if } \epsilon_{p}(g)=-1\end{cases}
$$

Let $K(g)=\frac{\alpha(g)}{f(g)}$ on $G_{v}$. Then the cocycle $c_{\alpha}$ can be decomposed as $c_{\alpha}=c_{K} c_{f}$. Clearly $K(i)$ belongs to $F_{v}^{*}$, for all $i \in I_{v}$. Using Lemma 9 applied to $K$ and $t=\epsilon^{\prime}$, we have

$$
\operatorname{inv}_{v}\left(c_{K}\right)=\frac{1}{2} \cdot v\left(\frac{K^{2}}{\epsilon^{\prime}}\left(\sigma_{v}\right)\right)=\frac{1}{2} \cdot m_{v}^{+} \bmod \mathbb{Z} .
$$

To compute $\operatorname{inv}_{v}\left(c_{f}\right)$, let $\sigma$ be the nontrivial element of $\operatorname{Gal}\left(F_{v}(\sqrt{t}) / F_{v}\right)$. Then the cocycle table of the cocycle $c_{f}$ is given by

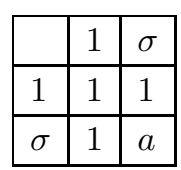

which gives the symbol $(t, a)_{v}$. This proves (1).

We now turn to parts (2) and (3). We wish to find $d \in F^{*}$, such that $\alpha(i) \equiv \sqrt{d} \bmod F_{v}^{*}$, if $\epsilon_{p}(i)=-1$. We cannot take $d=a$ in parts (2) and (3) since $a=0$ or $\infty$. So we argue a bit differently.

Let $i \in I_{v}$ with $\epsilon_{p}(i)=-1$. We claim that $\alpha(i) \equiv a_{p^{\dagger}} \bmod F^{*}$. By (3.1) and the proof of Theorem 16, if $\chi_{\gamma}$ is unramified at $p$, then $\alpha(i)^{\gamma}=\alpha(i)$. Similarly, if $\chi_{\gamma}$ is ramified at $p$, then $\alpha(i)^{\gamma}=\chi_{\gamma}(i) \alpha(i)=\epsilon_{p}(i) \alpha(i)=-\alpha(i)$. Thus, if Frob $_{p^{\dagger}}$ is an arithmetic Frobenius at the prime $p^{\dagger}$, then $\alpha(i) \equiv \alpha\left(\right.$ Frob $\left._{p^{\dagger}}\right) \equiv a_{p^{\dagger}}$ $\bmod F^{*}$, as claimed. Define $f$ on $G_{v}$ by

$$
f(g)= \begin{cases}1 & \text { if } \epsilon_{p}(g)=1 \\ a_{p^{\dagger}} & \text { if } \epsilon_{p}(g)=-1\end{cases}
$$

Let $K(g)=\frac{\alpha(g)}{f(g)}$ on $G_{v}$. Then the cocycle $c_{\alpha}$ can be decomposed as $c_{\alpha}=c_{K} c_{f}$. We now proceed as in the proof of part (1). If $a_{p}^{f_{v}}+\left(\bar{a}_{p} \epsilon^{\prime}(p)\right)^{f_{v}} \neq 0$, the cocycle $c_{K}$ has invariant $\operatorname{inv}_{v}\left(c_{K}\right)=\frac{1}{2} \cdot m_{v}^{+} \bmod \mathbb{Z}$. If $a_{p}^{f_{v}}-\left(\bar{a}_{p} \epsilon^{\prime}(p)\right)^{f_{v}} \neq 0$, then we get an extra term on evaluating $\alpha$ at an arithmetic Frobenius Frob ${ }_{v}$ for which 
$\epsilon_{p}\left(\operatorname{Frob}_{v}\right)=-1$, and get $\operatorname{inv}_{v}\left(c_{K}\right)=\frac{1}{2} \cdot\left(m_{v}^{-}-e_{v} \cdot v(b)\right)$. It remains to calculate $\operatorname{inv}_{v}\left(c_{f}\right)$. Let $\sigma$ be the nontrivial element of the Galois group of the quadratic field cut out by $\epsilon_{p}$. The table for the cocycle $c_{f}$ is given by

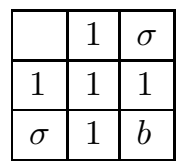

which is clearly the symbol $(t, b)_{v}$. This proves $(2)$ and $(3)$.

The above theorem shows that the ramification of $X$ at the place $v$ is determined by $m_{v}^{ \pm}$and one extra Hilbert symbol. We can calculate those symbols using the formulas of pages 211-212 of [Se80], except if $p=2$ and $F_{v} \neq \mathbb{Q}_{2}$, in which case we can use the formulas stated, e.g., in [Sn81] and [FV93].

\section{Supercuspidal primes}

We assume in this section that $p$ is an odd prime, $N_{p} \geq 2$ and $N_{p}>C_{p}$, and prove a weak result on the ramification of $X_{v}$. Since $a_{p}=0$, results of the kind proved so far, relating the ramification to the valuations of expressions involving the Fourier coefficients at $p$, are no longer possible.

Note that when the local Galois representation is a twist of cases already treated above we can often predict the ramification since the Brauer class of the endomorphism algebra is invariant under twist. Thus we may assume that the local Galois representation is supercuspidal, and is induced by a character $\chi$ of an index two subgroup $G_{K}$ of the local Galois group $G_{p}=G_{\mathbb{Q}_{p}}$, i.e.,

$$
\left.\rho_{f}\right|_{G_{p}} \sim \operatorname{Ind}_{G_{K}}^{G_{p}} \chi
$$

We manage to sometimes predict the ramification of $X_{v}$ in terms of this character. Let $\sigma$ be the non-trivial automorphism of $K / \mathbb{Q}_{p}$. We define an extension $L$ of $F_{v}$ and for an arithmetic Frobenius Frob $_{u}$ of $L$ set

$$
m_{v}:=e_{v} \cdot v\left(\frac{\left(\chi\left(\operatorname{Frob}_{u}\right)+\chi^{\sigma}\left(\operatorname{Frob}_{u}\right)\right)^{2}}{\epsilon^{\prime}(p)^{f_{v}}}\right) \in \mathbb{Z} \cup\{\infty\},
$$

where $v$ is normalized such that $v(p)=1$. Note

$$
m_{v}<\infty \text {, if } \chi\left(\operatorname{Frob}_{u}\right)+\chi^{\sigma}\left(\operatorname{Frob}_{u}\right) \neq 0 .
$$

Proposition 33: Assume $F_{v}$ contains $K$. If $m_{v}$ is finite, then $X_{v}$ is a matrix algebra over $F_{v}$ if and only if $m_{v}$ is even. 
Proof. Since $F_{v}$ contains $K$, we have

$$
\left.\rho_{f}\right|_{I_{v}} \sim\left(\begin{array}{cc}
\chi & 0 \\
0 & \chi^{\sigma}
\end{array}\right) .
$$

So if $i \in I_{v}$ and $\chi(i) \neq-\chi^{\sigma}(i)$, then by part (4) of Proposition 8

$$
\alpha(i) \equiv\left(\chi(i)+\chi^{\sigma}(i)\right) \bmod F^{*} .
$$

If $K=\mathbb{Q}_{p^{2}}$ is unramified, then we may write $\left.\chi\right|_{I_{K}}=\omega_{2}^{j} \chi_{1} \chi_{2}$, where, following the notation of [GM09, §3.3], $\omega_{2}$ is the fundamental character of level two and $\chi_{i}$, for $i=1,2$, are characters of $p$-power order. On the other hand, if $K / \mathbb{Q}_{p}$ is ramified then, in the notation of [GM09, §3.4], we may write $\left.\chi\right|_{I_{K}}=\omega^{j} \chi_{1} \chi_{2}$, where $\omega$ is the Teichmüller character and again the $\chi_{i}$ have $p$-power order. Choose an extension $L$ of odd degree over $F_{v}$ such that $\epsilon_{p}$ is tame and $\chi_{i}$, for $i=1,2$, are trivial, when restricted to $I_{L}$. In the unramified case, $\omega_{2}^{\sigma}=\omega_{2}^{p}$, and we get $\alpha(i) \equiv \omega_{2}^{j}(i)+\omega_{2}^{p j}(i) \bmod F^{*}$, for $i \in I_{L}$ such that the expression on the right is non-zero. Since $\omega_{2}$ takes values in the $\left(p^{2}-1\right)$-th roots of unity and $F_{v}$ contains $\mathbb{Q}_{p^{2}}$, we see that $\alpha(i) \in F_{v}^{*}$, for $i \in I_{L}$, under the non-vanishing assumption. Since, $\tilde{\alpha}$ is a homomorphism, we can show $\alpha(i)$ belongs to $F_{v}^{*}$ even if the expression on the right vanishes, by the usual argument. In the ramified case, $\omega=\omega^{\sigma}$, and we have $\alpha(i) \equiv\left(\omega^{j}(i)+\omega^{\sigma j}(i)\right)=2 \omega^{j}(i) \bmod F^{*}$, for $i \in I_{L}$. Since $\omega$ takes values in the $(p-1)$-th roots of unity, we again deduce that $\alpha(i) \in F_{v}^{*}$, for $i \in I_{L}$.

Let $u$ be the prime of $L$ lying over $v$. By Lemma 9 applied to $K=\alpha$ and $t=\epsilon^{\prime}$, both restricted to $G_{L}$, we have

$$
\operatorname{inv}_{L}\left(\operatorname{res}_{F_{v} \mid L} c_{\alpha}\right)=\frac{1}{2} \cdot u\left(\frac{\alpha^{2}}{\epsilon^{\prime}}\left(\operatorname{Frob}_{u}\right)\right) \bmod \mathbb{Z} .
$$

Here as usual, since $\epsilon_{p}$ is tame when restricted to $L, \epsilon_{p}(g) \in \mathbb{Q}_{p}^{*}$, and since $\frac{\alpha^{2}}{\epsilon}(g) \in F^{*}$, we get $\frac{\alpha^{2}}{\epsilon^{\prime}}(g) \in F_{v}^{*}$, for all $g \in G_{L}$. If $\left[L: F_{v}\right]=p^{t}$, then $\operatorname{inv}_{L}\left(\operatorname{res}_{F_{v} \mid L} c_{\alpha}\right)=p^{t} \cdot \operatorname{inv}_{v}\left(c_{\alpha}\right)$, so $X_{v}$ is a matrix algebra over $F_{v}$ if and only if $e_{L / F_{v}} \cdot v\left(\frac{\alpha^{2}}{\epsilon^{\prime}}\left(\right.\right.$ Frob $\left.\left._{u}\right)\right)$ is even, where $e_{L / F_{v}}=p^{t}$ is the degree of the totally ramified extension $L / F_{v}$ and $v$ is the surjective valuation of $F_{v}^{*}$ onto $\mathbb{Z}$. If we choose the valuation $v$ such that $v(p)=1$, then $X_{v}$ is a matrix algebra if and only if $e_{v} \cdot v\left(\frac{\alpha^{2}}{\epsilon^{\prime}}\left(\operatorname{Frob}_{u}\right)\right)$ is even. Since the inertia degree of $L / F_{v}$ is also $f_{v}$, we get the desired result. 


\section{Numerical examples}

We end this paper with some examples. For forms of quadratic nenbetypus, the examples were generated by the program Endohecke due to Brown and Ghate, which was made by suitably modifying the $\mathrm{C}++$ program Hecke created by W. Stein. The notation for the nebentypus is the one used in these programs (and not that used in [GGQ05]). For forms of non-quadratic nebentypus, we used tables of Quer [Qu05]. The first example is a Steinberg case, the next two are unequal slope ramified principal series (RPS) cases, whereas the remaining examples are equal slope RPS cases.

(1) Let $f \in S_{5}(15,[2,1])$ be the unique primitive form. It is Steinberg at the prime 5 since $N_{5}=1$ and $C_{5}=0 . F$ is a cubic extension of $\mathbb{Q}$. Now, 5 decomposes into two distinct primes $v_{1}, v_{2}$ in $F$ with ramification index and inertia degree $(1,1)$ and $(2,1)$ respectively. It turns out that $X_{v_{1}}$ is ramified but $X_{v_{2}}$ is not ramified, as predicted by Theorem 15 .

(2) Let $f \in S_{3}(35,[2,2])$ be the unique primitive form of orbit size $4 . F=\mathbb{Q}$ and $X$ is ramified at the RPS prime 5 . Also $v_{5}(\mu+\nu)=v_{5}(\mu+\nu+10)=$ 1, corroborating Theorem 22 .

(3) Let $f \in S_{2}(88,[2,2,2])$ be the unique primitive form of orbit size 4 . Then $F=\mathbb{Q}(\sqrt{2})$ and $X$ is ramified at the unique prime $v$ of $F$ lying above the RPS prime 2. One checks $\mu+\nu=-\sqrt{2}$ so $v(\mu+\nu)=$ $v(\mu+\nu+4)=1 / 2$, hence $m_{v}=\left[F_{v}: \mathbb{Q}_{2}\right] \cdot 1 / 2=1$ is odd, supporting Theorem 27.

(4) Let $f \in S_{2}(35,[4,2])$ be the unique primitive form of orbit size 4 . Then $F=\mathbb{Q}$ and $X$ is ramified at the RPS prime 5 (and also at the good prime 2). One checks $\mu=-\nu=-5 i$, so $v_{5}(\mu+\nu+10)=1$, hence $m_{v}^{+}=1$ is odd, supporting Theorem 28 .

(5) Let $f \in S_{2}(112,[2,4,2])$ be the primitive form of orbit size 4 with $a_{2}=$ $1+i$. Then $F=\mathbb{Q}$ and $p=2$ is an RPS prime, since $\epsilon_{2}$ has level 16, with 'tame' part of order 2 , and 'wild' part of order 4 . Now $\mu=-\nu=2 i$, so $v(\mu+\nu \pm 2 p)=v( \pm 4)=2$ is even, yet $X$ is ramified at $2(X$ is also ramified at 3). This interesting 'switch in parity' is predicted by part (2) of Corollary 31. We thank E. González-Jiménez for finding this example. 
(6) Let $f \in S_{2}(363,[2,2])$ be the unique eigenform of orbit size 4 . Then $F=\mathbb{Q}$ and $X$ is ramified at the RPS prime 3. Yet $m_{v}=m_{v}^{+}=$ $v_{3}(\mu+\nu+6)=2$ is even. This is an equal slope case, so we use Theorem 32 . We compute that $a=-3$ and $t=-3$. Since $(-3,-3)_{3}=-1$, we have $n_{v}=1 \bmod 2$. Thus part (1) of Theorem 32 holds, and explains the switch in parity.

(7) Let $f \in S_{3}(91,[2,2])$ be the eigenform of orbit size 4 . Then $F=\mathbb{Q}$ and $X$ is not ramified at the RPS prime 7 . Now $a_{7}= \pm 7 i=\bar{a}_{7} \epsilon^{\prime}(7)$, so we cannot use part (1) of Theorem 32, since $\mu=\nu=49$ and $a=\infty$. We use part (2) instead. We take $p^{\dagger}=3$ and see $b=a_{3}^{2}=-26$. Also, $t=-7$ and $m_{v}^{+}=2$ is even. We have $(-26,-7)_{7}=1$, so $n_{v}=0$. This corroborates part (2) of Theorem 32 .

ACKnowledgements. D. B. was partly supported by ARC grant DP0773301. He thanks the second author for constant encouragement during the course of his Ph.D. at TIFR. E.G. was partly supported by the Indo-French (CEFIPRAIFCPAR) project 3701-2. Part of this work was done at the Institut Henri Poincaré during the "Galois trimester" in 2010. Finally, the authors thank Ken Ribet for his interest and his support.

\section{References}

[AL78] A. Atkin and W. Li, Twists of newforms and pseudo-eigenvalues of $W$-operators, Inventiones Mathematicae 48 (1978), 221-243.

[BG11] D. Banerjee and E. Ghate, Crossed product algebras attached to weight one forms, Mathematical Research Letters 18 (2011), 141-150.

[BG04] A. Brown and E. Ghate, Endomorphism algebras of motives attached to elliptic modular forms, Université de Grenoble. Annales de l'Institut Fourier 53 (2003), $1615-1676$.

[FV93] I. Fesenko and S. Vostokov, Local Fields and Their Extensions. A Constructive Approach, Translations of Mathematical Monographs, Vol. 121, American Mathematical Society, Providence, RI, 1993.

[GJ78] S. Gelbart and H. Jacquet, A relation between automorphic representations of GL(2) and GL(3), Annales Scientifiques de l'École Normale Supérieure 11 (1978), 471-542.

[GGQ05] E. Ghate, E. González-Jiménez and J. Quer, On the Brauer class of modular endomorphism algebras, International Mathematics Research Notices 12 (2005), 701723.

[GM09] E. Ghate and A. Mézard, Filtered modules with coefficients, Transactions of the American Mathematical Society 361 (2009), 2243-2261. 
[Hi00] H. Hida, Modular Forms and Galois cohomology, Cambridge Studies in Advanced Mathematics, Vol. 69, Cambridge University Press, 2000.

[KZ91] W. Kohnen and D. Zagier, Values of L-series of modular forms at the center of the critical strip, Inventiones Mathematicae 64 (1981), 175-198.

[Mi89] T. Miyake, Modular Forms, Springer-Verlag, Berlin, 1989.

[Mo81] F. Momse, On the l-adic representations attached to modular forms, Journal of the Faculty of Science. University of Tokyo. Section IA Mathematics 28 (1981), 89-109.

[Pi98] R. Pink, Compact subgroups of linear algebraic groups, Journal of Algebra 206 (1998), 438-504.

[Qu98] J. Quer, La classe de Brauer de l'algèbre d'endomorphismes d'une variété abélienne modulaire, Comptes Rendus de l'Académie des Sciences 327 (1998), 227-230.

[Qu05] J. Quer, Tables of modular endomorphism algebras $X_{f}=\operatorname{End}\left(M_{f}\right)$ of motives $M_{f}$ attached to non-CM newforms, 255 pages, 2005.

[Ri75] K. Ribet, Endomorphisms of semi-stable abelian varieties over number fields, Annals of Mathematics 101(1975), 555-562.

[Ri80] K. Ribet, Twists of modular forms and endomorphisms of abelian varieties, Mathematische Annalen 253 (1980), 43-62.

[Ri81] K. Ribet, Endomorphism algebras of abelian varieties attached to newforms of weight 2, Progress in Mathematics, Vol. 12, Birkhäuser, Boston, MA, 1981, pp. 263276 .

[Ri85] K. Ribet, On $\ell$-adic representations attached to modular forms. II, Glasgow Mathematical Journal 27 (1985), 185-194.

[Ri04] K. Ribet, Abelian varieties over $\mathbb{Q}$ and modular forms, in Modular Curves and Abelian Varieties, Progress in Mathematics, Vol. 224, Birkhaüser, Basel, 2004, pp. 241-261.

[Sa97] T. Saito, Modular forms and p-adic Hodge theory, Inventiones Mathematicae 129 (1997), 607-620.

[Sh71] G. Shimura, Introduction to the Arithmetic Theory of Automorphic Functions, Princeton University Press, Princeton, 1971.

[Sh73] G. Shimura, On modular forms of half integral weight, Annals of Mathematics 97 (1973), 440-481.

[Sc90] A. Scholl, Motives for modular forms, Inventiones Mathematicae 100 (1990), 419430 .

[Sn81] S. Sen, On explicit reciprocity laws. II, Journal für die Reine und Angewandte Mathematik 323(1981), 68-87.

[Se80] J.-P. Serre, Local Fields, Graduate Texts in Mathematics, Vol. 67, Springer-Verlag, Berlin, 1980.

[Wa81] J.-L. Waldspurger, Sur les coefficients de Fourier des formes modulaires de poids demi-entier, Journal de Mathématiques Pures et Appliquées 9 (1981), 375-484. 